\title{
Conformal mapping and shot noise in graphene
}

\author{
Adam Rycerz, ${ }^{1,2}$ Patrik Recher, ${ }^{3}$ and Michael Wimmer ${ }^{2, *}$ \\ ${ }^{1}$ Marian Smoluchowski Institute of Physics, Jagiellonian University, Reymonta 4, PL-30059 Kraków, Poland \\ ${ }^{2}$ Institut für Theoretische Physik, Universität Regensburg, D-93040 Regensburg, Germany \\ ${ }^{3}$ Institut für Theoretische Physik und Astrophysik, Universität Würzburg, Am Hubland, D-97074 Würzburg, Germany
}

(Received 12 June 2009; revised manuscript received 17 August 2009; published 18 September 2009)

\begin{abstract}
Ballistic transport through a collection of quantum billiards in undoped graphene is studied analytically within the conformal mapping technique. The billiards show pseudodiffusive behavior, with the conductance equal to that of a classical conductor characterized by the conductivity $\sigma_{0}=4 e^{2} / \pi h$ and the Fano factor $F$ $=1 / 3$. By shrinking at least one of the billiard openings, we observe a tunneling behavior, where the conductance shows a power-law decay with the system size, and the shot noise is Poissonian $(F=1)$. In the crossover region between tunneling and pseudodiffusive regimes, the conductance $G \approx(1-F) \times s e^{2} / h$. The degeneracy $s=8$ for the Corbino disk, which preserves the full symmetry of the Dirac equation, $s=4$ for billiards bounded with smooth edges which break the symplectic symmetry, and $s=2$ when abrupt edges lead to strong intervalley scattering. An alternative, analytical, or numerical technique is utilized for each of the billiards to confirm the applicability of the conformal mapping for various boundary conditions.
\end{abstract}

DOI: $10.1103 /$ PhysRevB.80.125417

PACS number(s): 73.50.Td, 73.23.Ad, 73.63.-b

\section{INTRODUCTION}

The isolation of single layers of carbon (graphene) whose low-energy spectrum is described by the Dirac-Weyl Hamiltonian of massless spin-1/2 fermions ${ }^{1}$ has offered physicists the unique possibility to test the predictions of relativistic quantum mechanics in a condensed phase. A particular attention focuses on ballistic transport, ${ }^{2}$ as the unusual band structure of a carbon monolayer ${ }^{3}$ leads simultaneously to a divergent Fermi wavelength $\lambda_{F} \rightarrow \infty$ in the undoped graphene limit, and to a zero band gap. For these reasons, the quantum-mechanical wave character of an electron plays an essential role in transport even through a macroscopic graphene sample provided that the influence of disorder is negligible. ${ }^{4}$ A separate issue concerns the fact that Dirac fermions in graphene occur in two degenerate families, resulting from the presence of two different valleys in the band structure. This valley degree of freedom offers conceptually new possibilities to control charge carriers: the so-called "valleytronics." 5

So far, extensive theoretical studies of ballistic transport based on mode-matching analysis for the Dirac equation ${ }^{6,7}$ are available for a rectangular graphene sample of width $\mathcal{W}$, length $\mathcal{L}$, and various types of boundary conditions (bc). In the regime of large aspect ratios $\mathcal{W} / \mathcal{L} \gg 1$, the conductance of an undoped sample scales as $G=\sigma_{0} \mathcal{W} / \mathcal{L}$, with the universal conductivity $\sigma_{0}=4 e^{2} / \pi h$, regardless of boundary conditions. $^{8}$ Moreover, as shown by Tworzydło et al., ${ }^{7}$ the Fano factor in this case coincides with that of a diffusive wire $(F=1 / 3)$. Also, the transmission eigenvalues of these two systems display the same distribution. This analogy coined the term of pseudodiffusive transport, which describes ballistic graphene properties in the universal conductivity limit.

Recent experiments report an agreement with the theoretical predictions of Refs. 6 and 7 for either the conductance ${ }^{9}$ or the Fano factor. ${ }^{10}$ Furthermore, the temperature dependence of the conductivity ${ }^{11}$ also shows an approximate agreement with the ballistic theory generalized to finite temperatures. ${ }^{12}$ However, even for low temperatures, the convergence with $\mathcal{W} / \mathcal{L} \rightarrow \infty$ is much slower than predicted. In particular, for the largest aspect ratio $\mathcal{W} / \mathcal{L}=24$ studied in Ref. 10, the deviations from the limiting values $G \mathcal{L} / \mathcal{W}=\sigma_{0}$ and $F=1 / 3$ are close to $10 \%$, whereas results of Ref. 7 show the convergence should be already reached for moderate aspect ratios $\mathcal{W} / \mathcal{L} \gtrsim 4$. A clear explanation of this discrepancy is missing, but it is usually attributed to the fact that boundary conditions used in theoretical works, which describe either an abrupt termination of a perfect honeycomb lattice or an infinite-mass confinement, ${ }^{13}$ may not model the realsample edges correctly. ${ }^{14}$

In this work, we consider ballistic graphene systems of geometries for which the boundary effects are absent or suppressed. The paper is organized as follows. In Sec. II we briefly recall the mode-matching analysis for a graphene strip and show how to employ the conformal symmetry of the Dirac equation for undoped graphene ${ }^{15}$ to obtain analytically the transmission eigenvalues for other systems. Then, in Sec. III the method is applied to the Corbino disk. The results are compared with those obtained by direct mode matching for angular momentum eigenstates; a relation with the nonrelativistic electron gas in the disk setup is also discussed. In Sec. IV we study two basic billiards bounded with mass confinement: a finite section of the Corbino disk and a quantum dot with circular edges. The results obtained with the conformal mapping technique are confirmed by the computer simulation of transport using the tight-binding model on a honeycomb lattice. Finally, in Sec. V we study numerically the transport across an infinitely long nanoribbon by utilizing the four-terminal recursive Green's function algorithm, ${ }^{16}$ as well as across a finite section of a nanoribbon with an abrupt lattice termination. All the systems show pseudodiffusive transport behavior in a wide range of geometrical parameters. A further analogy between them appears when (at least) one of the leads is narrow in comparison to the characteristic length of the conducting region $L$. Namely, the conductance in such a quantum-tunneling regime shows 
a power-law decay $G \propto L^{-\alpha}$, where $\alpha$ is a nonuniversal (geometry dependent) exponent. Moreover, it is related to the shot noise by $F \approx 1-G h /\left(s e^{2}\right)$, so that the Poissonian limit $(F=1)$ is approached for large $L$. The symmetry-dependent factor $s=8$ in the presence of full spin, valley, and symplectic degeneracy (the case of the Corbino disk), or $s=4$ when the mass confinement, breaks symplectic symmetry of the Dirac equation. A final reduction to $s=2$ may be reached by adding abrupt (i.e., armchair) edges, which scatter carriers between the valleys.

The original feature of the geometries studied in this paper is that the influence of sample edges is eliminated (for the Corbino setup) or irrelevant, as the spatial current distribution is not uniform, but concentrated far away from the edges. This is why we believe that our theoretical findings could be confirmed experimentally with better precision than that for rectangular samples, as the role of boundary conditions is strongly suppressed.

\section{TRANSPORT OF DIRAC FERMIONS AND CONFORMAL MAPPING}

The compact derivation of transmission eigenvalues of a weakly doped (or undoped) graphene sample coupled to heavily doped graphene leads is known due to Sonin, ${ }^{17}$ who pointed out that one can first calculate the reflection and transmission amplitudes for an interface between weakly doped and heavily undoped regions and then employ the double-contact formula. ${ }^{18}$ Here we show that the derivation of Ref. 17 can be easily adopted to the Corbino disk, a finite section of it, and to a quantum dot with circular edges (all shown in Fig. 1), as these systems can be obtained from a strip by appropriate conformal transformations.

\section{A. Mode matching for a graphene strip}

Let us first consider an electron crossing from the weakly doped region $(x>0)$ to the heavily doped one $(x<0)$, as depicted in Fig. 2. The Dirac Hamiltonian for graphene has the well-known form ${ }^{19}$

$$
H_{0}=v_{F} \boldsymbol{\sigma} \cdot \boldsymbol{p},
$$

where $v_{F}$ is the Fermi velocity, $\boldsymbol{\sigma}=\left(\sigma_{x}, \sigma_{y}\right)$ is the vector operator build of Pauli matrices for the sublattice-pseudospin degree of freedom, and $\boldsymbol{p}=-i \hbar\left(\partial_{x}, \partial_{y}\right)$ is the in-plane momentum operator. Due to translational invariance along the $y$ axis, the solution of the Dirac equation with energy $E$ $=\hbar v_{F} k$ may be written as $\Psi(x, y)=\chi_{\theta}(x) e^{i k_{y} y},{ }^{20}$ with the transverse momentum $k_{y}=K_{y}\left(k_{i}\right.$ and $K_{i}$ with $i=x, y$ denote momentum components in the weakly and heavily doped regions, respectively), and the spinor

$$
\chi_{\theta}(x)=\left\{\begin{array}{cc}
\left(\begin{array}{c}
1 \\
-e^{-i \theta}
\end{array}\right) e^{-i k_{x} x}+r_{1}\left(\begin{array}{c}
1 \\
e^{i \theta}
\end{array}\right) e^{i k_{x} x}, & x>0 \\
t_{1} \sqrt{\frac{k_{x}}{k}}\left(\begin{array}{c}
1 \\
-1
\end{array}\right) e^{-i K_{x} x}, & x<0,
\end{array}\right.
$$

where $e^{i \theta}=\left(k_{x}+i k_{y}\right) / k$, and the limit of an infinite doping $(k \ll K)$ in the region $x<0$ is imposed. The continuity of the
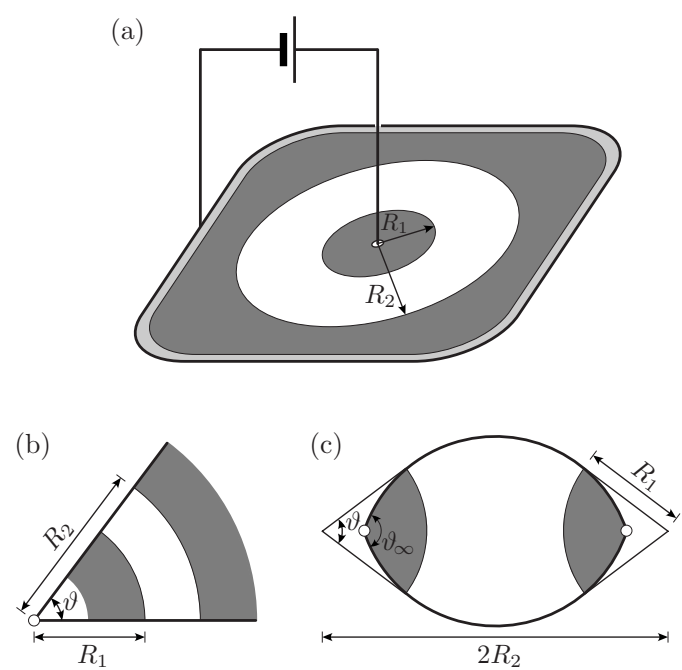

FIG. 1. Quantum billiards in undoped graphene studied analytically (schematic). (a) The Corbino disk with inner radius $R_{1}$ and outer radius $R_{2}$. (b) Generic section of the disk characterized by the spanning angle $\vartheta$. (c) Quantum dot with circular edges. A voltage source shown on panel (a) only drives the current through each of the devices. Shadow areas on all panels mark heavily doped (so highly conducting) graphene leads; white dots are the poles of conformal transformation mapping a given system onto a strip of Fig. 2. Thick lines on panels (b) and (c) indicate infinite-mass confinement.

two spinor components on both sides of the interface leads to expressions for the reflection and transmission amplitudes

$$
r_{1}=\frac{e^{-i \theta}-1}{e^{i \theta}+1}, \quad t_{1}=\frac{2 \sqrt{\cos \theta}}{e^{i \theta}+1} .
$$

The amplitudes $r_{1}$ and $t_{1}$ depend solely on the angle of incidence $\theta$ (see Fig. 2), illustrating the generic feature of ballistic transport in graphene that is insensitive to the lead details. ${ }^{21}$ The reflection and transmission amplitudes for an electron crossing from the undoped region to the second heavily doped lead are $r_{2}=r_{1}^{\star}$ and $t_{2}=t_{1}^{\star}$ (up to a phase factor), as the angle of incidence $\theta \rightarrow-\theta$ in this case. Thus, employing the double-contact formula of Ref. 18, the total transmission probability for phase-coherent transport through the system of Fig. 2 is

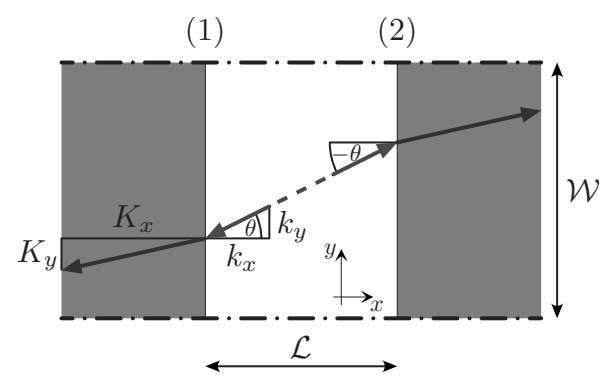

FIG. 2. Scattering on interfaces (1) and (2) between weakly doped (white area) and heavily doped (shaded area) regions in graphene. Horizontal dashed-dot lines mark symbolically generic boundary conditions applied to a strip. 


$$
T=\frac{\left|t_{1} t_{2}\right|^{2}}{\left|1-r_{1} r_{2} e^{2 i \phi_{12}}\right|^{2}}=\frac{1}{1+\left(\tan \theta \sin \phi_{12}\right)^{2}},
$$

where $\phi_{12} \equiv \int_{1}^{2} k_{x} d x$ is the phase shift earned by an electron when passing from the first interface to the second one. The above expression holds true for either propagating modes (for which $k_{x}=\sqrt{k^{2}-k_{y}^{2}}$ ) or, as an analytic continuation, for evanescent modes $\left(k_{x}=i q_{x}\right.$, with $\left.q_{x}=\sqrt{k_{y}^{2}-k^{2}}\right)$. For a confined geometry, the quantization of transverse momenta is determined by bc. ${ }^{6,7}$ Namely, $k_{y}=k_{y}^{l}$ (with $l$ integer) may be written in a compact form,

$$
k_{y}^{l}=\frac{g \pi(l+\gamma)}{\mathcal{W}},
$$

where $g=1,2$ for the closed and (generalized) periodic bc, respectively. $\gamma=\frac{1}{2}$ for either mass confinement or antiperiodic bc studied in this paper. (For a nanotubelike geometry as considered in Ref. 6, $\gamma=0$ corresponds to periodic bc.)

\section{B. Transmission via evanescent modes}

Here we limit ourselves to the case of undoped graphene $(k \rightarrow 0)$, in which the charge transport is carried fully by evanescent modes. An analytic continuation yields $\tan \theta \rightarrow i$ and $\phi_{12} \rightarrow i g \pi j \mathcal{L} / \mathcal{W}$ in Eq. (4), where we use the quantization (5) and define the half-integer $j \equiv l+\frac{1}{2}$. As pointed out by Katsnelson and Guinea, ${ }^{15}$ the zero-energy solution of the Dirac equation may be obtained via conformal transformation that links the considered geometry to a simple one, for which the wave function is known. ${ }^{22}$ In particular, if the conformal transformation $z(w)$ turns the system under consideration into a rectangle of width $\mathcal{W}$ and length $\mathcal{L}$ (Fig. 2), the transmission probability for the $j$ th evanescent mode may be written as

$$
T_{j}=\frac{1}{\cosh ^{2}[g j \ln \Lambda\{z(w)\}]}=\frac{4}{\left(\Lambda^{g j}+\Lambda^{-g j}\right)^{2}},
$$

where $j= \pm \frac{1}{2}, \pm \frac{3}{2}, \ldots$ (with the degeneracy $T_{j}=T_{-j}$ ). Notice that the amplitudes [Eq. (3)] remain unchanged after applying an arbitrary conformal transformation to the coordinate system of Fig. 2, so the only term in Eq. (4) affected by the transformation $z(w)$ is the phase shift $\phi_{12} \rightarrow i g j \ln \Lambda$. The real functional $\Lambda\{z(w)\}$ is defined by

$$
\ln \Lambda\{z(w)\} \equiv \pi \mathcal{L} / \mathcal{W}
$$

The explicit form of $\Lambda\{z(w)\}$ depends on the geometry and is given below for the examples of conformal transformation $z(w)$ having one and two poles in a complex plane, which allows us to obtain expressions for transmission probabilities through a finite section of the Corbino disk and through a quantum dot with circular edges, respectively.

But first, we discuss the two basic physical regimes of quantum transport in graphene, which are described by opposite limits of Eq. (6). The conductance of undoped graphene $^{6,7}$ is given by the Landauer formula

$$
G=\frac{s e^{2}}{h} \sum_{j=1 / 2,3 / 2, \ldots} T_{j}=s \pi \sigma_{0} \sum_{j}\left(\Lambda^{g j}+\Lambda^{-g j}\right)^{-2},
$$

with the degeneracy $s=4$ (spin and valley) for smooth mass confinement, and $s=8$ for antiperiodic bc due to an additional (symplectic) symmetry. ${ }^{23}$ The universal conductivity is $\sigma_{0} \equiv 4 e^{2} / \pi h$. The Fano factor also follows from summing over the modes

$$
F=\frac{\sum_{j=1 / 2,3 / 2, \ldots} T_{j}\left(1-T_{j}\right)}{\sum_{j=1 / 2,3 / 2, \ldots} T_{j}}
$$

but is affected by the symmetry-dependent factors $(g, s)$ only via $T_{j}-\mathrm{s}$ [Eq. (6)].

For the limit $\ln \Lambda \ll 1$, we can replace summation in Eq. (8) by integration and get

$$
G \approx G_{\mathrm{diff}}=\frac{\pi \sigma_{0}}{\ln \Lambda\{z(w)\}},
$$

where we use the relation $s=4 g$, valid for the two classes of bc studied here. In the $\ln \Lambda \ll 1$ limit, the relevant information about transmission probabilities is given by their statistical distribution,

$$
\rho(T)=\frac{2}{T \sqrt{1-T}} \frac{G_{\mathrm{diff}}}{\pi \sigma_{0}} .
$$

As the distribution $\rho(T)$ coincides with the known distribution $^{24}$ for diffusion modes in a disordered metal, $\ln \Lambda \ll 1$ constitutes a pseudodiffusive regime of transport through graphene billiards. Notice that the generic conformal transformation $z(w)$ affects $\rho(T)$ only via the prefactor $G_{\text {diff. }}$. In particular, the Fano factor

$$
F=1-\frac{\left\langle T^{2}\right\rangle}{\langle T\rangle} \approx \frac{1}{3},
$$

regardless of the particular form of $z(w)$. This observation may also help to understand why experimental results ${ }^{10}$ generally show better agreement with theory for the Fano factor rather than for the conductance. For instance, various geometrical defects (such as a corrugation of the lead-graphene interface) may affect $G_{\text {diff }}$ strongly but not affect $F$ at all.

In the opposite limit (ln $\Lambda \gg 1$ ), we find from Eq. (6) that $T_{1 / 2} \gg T_{3 / 2} \gg \ldots$, leading to

$$
G \approx s \pi \sigma_{0} \Lambda^{-g}, \quad F \approx 1-G \frac{h}{s e^{2}} .
$$

These expressions constitute a quantum-tunneling regime for ballistic graphene, in which the transport is governed by a single electronic mode with the fourfold (spin and valley) degeneracy. Below, we provide examples illustrating how the power-law dependence of $G$ on $\Lambda$ may be followed by a power-law decay of $G$ with the characteristic length scale of the system. 


\section{APPLICATION TO THE CORBINO DISK}

The Corbino setup, in which the graphene sample formed as an annulus is attached to coaxial leads, as shown schematically in Fig. 1(a), seems to be the simplest way to eliminate boundary effects, which are claimed to strongly affect experimental results for rectangular samples with small and moderate aspect ratios. ${ }^{9,10}$ In this section, we first utilize the conformal mapping technique to find transmission eigenvalues for an undoped disk and then compare the results with that obtained by a direct wave-function matching, possible also for a doped disk.

\section{A. Conformal mapping for an undoped disk}

The conformal transformation that changes the Corbino disk with the inner radius $R_{1}$ and the outer radius $R_{2}$ shown in Fig. 1(a) into a rectangle of the width $\mathcal{W}$ and the length $\mathcal{L}$ (see Fig. 2) is given by ${ }^{25}$

$$
z=\frac{\mathcal{W}}{2 \pi} \log \frac{w}{R_{1}} .
$$

(Hereinafter, we use the symbol log to denote the natural logarithm in a complex domain.) For the complex variable $z=x+i y$, with $0 \leq x \leq \mathcal{L}$, and $0 \leq y \leq \mathcal{W}$, transformation (14) leads to $R_{1} \leq|w| \leq R_{2}$ and $0 \leq \arg w \leq 2 \pi$ provided the condition $R_{2} / R_{1}=e^{2 \pi \mathcal{L} / \mathcal{W}}$ is satisfied. Using Eq. (7), such a condition implies the functional $\Lambda\{z(w)\}$ to have the form

$$
\Lambda=\Lambda\left(R_{1}, R_{2}\right)=\left(\frac{R_{2}}{R_{1}}\right)^{1 / 2} .
$$

As the conformal mapping is known, the only part to be explained now is the boundary conditions applied to a strip of Fig. 2. To define them, one needs to notice that after a rotation by $2 \pi$ in the coordinate system of Fig. 1(a), the spinor part of the wave function acquires the Berry phase $\mathrm{e}^{26,27}$ $e^{i \pi \sigma_{z}}=-1$. Within the mapping (14), a rotation by $2 \pi$ turns into a shift along the $y$ axis in Fig. 2 by a strip width $\mathcal{W}$. This is why the spinor-rotational invariance of the original wave function implies antiperiodic boundary conditions $\Psi(x, y$ $+\mathcal{W})=-\Psi(x, y)$ for a strip. Such boundary conditions, together with the functional $\Lambda\{z(w)\}$ given by Eq. (15), lead the formula (6) for transmission probabilities to a form

$$
T_{j}=\frac{1}{\cosh ^{2}\left[j \ln \left(R_{1} / R_{2}\right)\right]}, \quad j=\frac{1}{2}, \frac{3}{2}, \frac{5}{2}, \ldots .
$$

A generalization for the setup with circular but not coaxial contacts is presented in Appendix A.

The dependence of the conductance (8) and the Fano factor (9) on the radii ratio $R_{1} / R_{2}$ is plotted in Fig. 3 (solid lines). The limiting behavior for $R_{1} / R_{2} \approx 1$, corresponding $\ln \Lambda \ll 1$ [Eq. (15)] is characterized by $G \approx G_{\text {diff }}$ [Eq. (10)], with

$$
G_{\mathrm{diff}}=\frac{2 \pi \sigma_{0}}{\ln \left(R_{2} / R_{1}\right)}, \quad F \approx \frac{1}{3} .
$$

The formula for $G_{\text {diff }}$ coincides with the well-known classical conductance of the Corbino disk. ${ }^{28}$ The asymptotic values

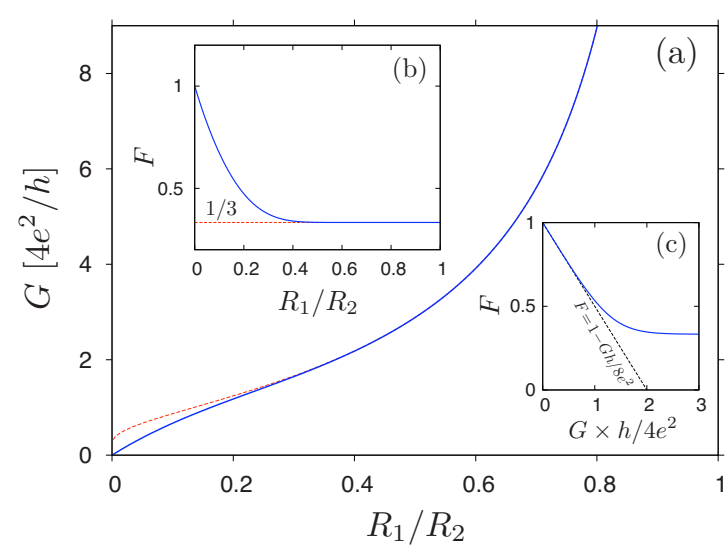

FIG. 3. (Color online) Conductance and Fano factor for the undoped Corbino disk in graphene, as a function of the radii ratio [(a) and (b)] and the shot noise vs (c) conductance diagram. The curves calculated from Eqs. (8) and (9) are plotted with solid lines on all panels. Dashed lines show the pseudodiffusive limit (17) on panels (a) and (b) and the tunneling limit (18) on panel (c).

[Eq. (17)] are depicted with dashed red lines on Figs. 3(a) and 3(b). In the opposite limit $\left(R_{1} \ll R_{2}\right)$, Eq. (13) takes the form

$$
G \approx 8 \pi \sigma_{0} \frac{R_{1}}{R_{2}}, \quad F \approx 1-G \frac{h}{8 e^{2}} .
$$

The second formula from above is shown in Fig. 3(c) with a dashed black line.

The results presented in Fig. 3 show that the pseudodiffusive formulas [Eq. (17)] for $G$ and $F$ match the exact expressions (8) and (9) with $T_{j}$ given by Eq. (16) in a relatively wide range of ratios $R_{1} / R_{2}$. Namely, the agreement becomes better than $1 \%$ if $R_{1} \geq 0.29 R_{2}$ for the conductance and if $R_{1}$ $\geq 0.43 R_{2}$ for the Fano factor. For smaller $R_{1} / R_{2}$, one can identify the crossover from the pseudodiffusive to quantumtunneling behavior. In particular, the exact values of $G$ are closer to the tunneling formula (18) than to the pseudodiffusive formula (17) below $R_{1} / R_{2}=0.11$. The same is observed for $F$ below $R_{1} / R_{2}=0.16$. The most characteristic feature of the tunneling regime is the relation $G \approx(1-F) \times 8 e^{2} / h$, following from Eq. (18). It is satisfied with an accuracy better than $10 \%$ for $G \leqq 4 e^{2} / h$ (or $F \gtrsim 0.5$ ), corresponding to $R_{1} / R_{2} \leqq 0.2$. In this range, we also find that the conductance decays (at fixed $R_{1}$ ) as $G \propto 1 / L$, where $L \equiv R_{2}-R_{1} \approx R_{2}$ is the characteristic length of the sample area.

A similar power-law decay of the conductance with the sample length is predicted for geometries with noncoaxial leads considered in Appendix A. In the two limiting situations, the Möbius transformation (A1) maps an infinite plane (hemiplane) with two (one) narrow circular openings onto the Corbino disk. Physically, these two situations correspond to the setup consisting of two circular leads probing a large graphene sample [see Fig. 4(a)] and of one circular lead and a long straight interface between the undoped and the heavily doped region playing the role of a second lead [see Fig. 4(b)]. In the first case, the mapping (A1) leads to $\Lambda \approx l / r$ and, subsequently, to the quadratic decay of the conductance 

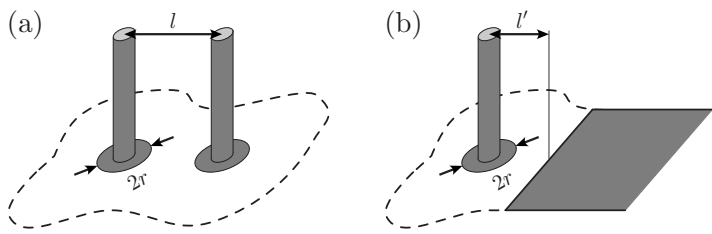

FIG. 4. Large graphene flake probed by two circular leads of radius $r$ separated by the distance (a) $l$ and by a lead placed in the distance $l^{\prime}$ from a straight interface between undoped and heavily doped regions (b).

$$
G \approx 8 \pi \sigma_{0}\left(\frac{r}{l}\right)^{2} \quad \text { for } \quad r \ll l
$$

(with the radius of each lead $r$ and the distance between leads $l)$. In the second case, the functional $\Lambda \approx\left(2 l^{\prime} / r\right)^{1 / 2}$ and the conductance

$$
G \approx 4 \pi \sigma_{0} \frac{r}{l^{\prime}}
$$

shows reciprocal decay with the sample area length, similarly as observed for the Corbino disk. The approximate relation between the conductance and the Fano factor $G \approx(1-F) \times 8 e^{2} / h$ holds true for both situations of Fig. 4 , showing the tunneling-transport regime in graphene appears generically for a setup consisting of (at least) one narrow circular lead.

\section{B. Electron transport at finite doping}

We complement the study of the Corbino disk in graphene with its transport properties at finite doping characterized by the chemical potential $\mu_{0} \equiv \pm \hbar v_{F} k$ (where $\mu_{0}>0$ and $\mu_{0}$ $<0$ refers to electron and hole doping, respectively). The analysis is closely related to that for the electronic levels of graphene rings. ${ }^{29}$ The single valley Hamiltonian for the doped disk reads as

$$
H=H_{0}+U(r) \sigma_{0}
$$

where $H_{0}$ is given by Eq. (1), the electrostatic potential $U(r)=U_{0}$ if $R_{1} \leq r \leq R_{2}$, and $U(r)=U_{\infty}$ otherwise. The chemical potential $\mu_{0}=E-U_{0}$ in the disk or $\mu_{\infty}=E-U_{\infty}$ in the leads. The rotational invariance of the problem allows us to perform the mode matching for each eigenstate of the total angular momentum $J_{z}=l_{z}+\hbar \sigma_{z} / 2$ (with $l_{z} \equiv-i \hbar \partial_{\varphi}$ the orbital angular momentum) separately. The eigenstate of the Hamiltonian (21) corresponding to the $j$ th eigenvalue of $J_{z}$ can be written as

$$
\psi_{j}(r, \varphi)=e^{i(j-1 / 2) \varphi}\left(\begin{array}{c}
\chi_{j, A}(r) \\
\chi_{j, B}(r) e^{i \varphi}
\end{array}\right)=e^{i\left(j-\sigma_{z} / 2\right) \varphi} \chi_{j}(r)
$$

where $j$ is a half-odd integer $j= \pm \frac{1}{2}, \pm \frac{3}{2}, \ldots$ For the electron doping $[E-U(r)>0]$, the radial components $\chi \equiv\left[\chi_{A}, \chi_{B}\right]^{T}$ for the incoming and outgoing waves are given (up to the normalization) by

$$
\chi_{j}^{\text {in }}=\left(\begin{array}{c}
H_{j-1 / 2}^{(2)}(\rho) \\
i H_{j+1 / 2}^{(2)}(\rho)
\end{array}\right), \quad \chi_{j}^{\text {out }}=\left(\begin{array}{c}
H_{j-1 / 2}^{(1)}(\rho) \\
i H_{j+1 / 2}^{(1)}(\rho)
\end{array}\right),
$$

where $H_{\nu}^{(1,2)}(\rho)$ is the Hankel function of the (first and second) kind, and the dimensionless radial coordinate is $\rho=\mid E$ $-U(r) \mid r /\left(\hbar v_{F}\right)[$ so $\rho=k r$ in the disk and $\rho=K r$ in the leads, with $\left.K \equiv\left|\mu_{\infty}\right| /\left(\hbar v_{F}\right)\right]$. The radial current density is $j_{r}$ $=e v_{F}\left\langle\psi_{j}^{\text {in(out) }}\left|\sigma_{r}\right| \psi_{j}^{\text {in(out })}\right\rangle= \pm 4 e v_{F} /(\pi \rho)$, where the upper (lower) $\operatorname{sign}$ is for $\psi_{j}^{\text {in }}\left(\psi_{j}^{\text {out }}\right), \sigma_{r}=\sigma_{x} \cos \varphi+\sigma_{y} \sin \varphi$, and we use the identity $\operatorname{Im}\left[H_{\nu}^{(1)}(\rho) H_{\nu+1}^{(2)}(\rho)\right]=2 /(\pi \rho)$. For the hole doping $[E-U(r)<0]$, the wave functions are $\tilde{\chi}_{j}^{\text {in }(\text { out })}$ $=\left[\chi_{j}^{\text {in (out) }}\right]^{\star}$, where we use the relation $H_{\nu}^{(2)}=\left[H_{\nu}^{(1)}\right]^{\star}$. The transmission and reflection amplitudes are obtained by the wave-function matching at $r=R_{1}$ and $r=R_{2}$. [Note that the $\varphi$ dependence of the spinor (22) plays no role for the modematching analysis.]

Details of the calculations are given in Appendix B. For $\left|\mu_{\infty}\right| \rightarrow \infty$ (the heavily doped leads limit), the transmission probability for the $j$ th mode $T_{j}=T_{j}\left(\mu_{0}\right)$ reads as

$$
T_{j}=\frac{16}{\pi^{2} k^{2} R_{1} R_{2}} \frac{1}{\left(\mathfrak{D}_{j}^{+}\right)^{2}+\left(\mathfrak{D}_{j}^{-}\right)^{2}},
$$

with

$$
\mathfrak{D}_{j}^{ \pm}=\operatorname{Im}\left[H_{j-1 / 2}^{(1)}\left(k R_{1}\right) H_{j \mp 1 / 2}^{(2)}\left(k R_{2}\right) \pm H_{j+1 / 2}^{(1)}\left(k R_{1}\right) H_{j \pm 1 / 2}^{(2)}\left(k R_{2}\right)\right] .
$$

Equations (8) and (9) for $G$ and $F$ remain unchanged since we again observe the symmetry $T_{-j}=T_{j}$. In addition, the particle-hole symmetry $T_{j}\left(-\mu_{0}\right)=T_{j}\left(\mu_{0}\right)$ allows us to limit the discussion to $\mu_{0}>0$.

Numerical values for the conductance and Fano factor of the doped disk are presented in Fig. 5. Following the idea of Kirczenow, ${ }^{30}$ we compare [in Fig. $5(\mathrm{a})$ ] the exact quantum conductance given by Eqs. (8) and (24) with the semiclassical approximation for large angular momenta

$$
G_{\mathrm{s}-\mathrm{cl}}=\frac{8 e^{2}}{h}\left(j_{1}+\frac{1}{2}\right),
$$

where $j_{1}=\operatorname{int}\left(k R_{1}-\frac{1}{2}\right)+\frac{1}{2}$ is the maximal value of $j$ such that $j \leq k R_{1}$. Surprisingly, the quantization steps of $G_{\mathrm{s}-\mathrm{cl}}$ (dotted black line) are missing in the actual data even for an extremely small radii ratio (solid blue and dashed red line for $R_{1} / R_{2}=0.1$ and 0.5 , respectively). Instead, weak modulation with a period $\approx \pi \hbar v_{F} /\left(R_{2}-R_{1}\right)$ is observed when varying $\mu_{0}$. Earlier, conductance quantization (with the steps of $4 e^{2} / h$ ) was predicted to appear for a graphene strip with a moderate aspect ratio $\mathcal{W} / \mathcal{L} \lesssim 1 .^{5,8}$ The quantization with the steps of $8 e^{2} / h$ was found theoretically for a bipolar junction in graphene, which shows the Goos-Hänchen effect. ${ }^{31}$ The lack of conductance quantization observed here for the Corbino disk shows that the role of evanescent modes, showing a slow (power-law) decay with distance, is also crucial far away from the Dirac point, illustrating a striking consequence of angular momentum conservation.

Similar to the strip geometry, ${ }^{7}$ the conductance minimum at $\mu_{0}=0$ corresponds to the maximum of the Fano factor [see Figs. 5(b)]. The peak width shrinks approximately as 

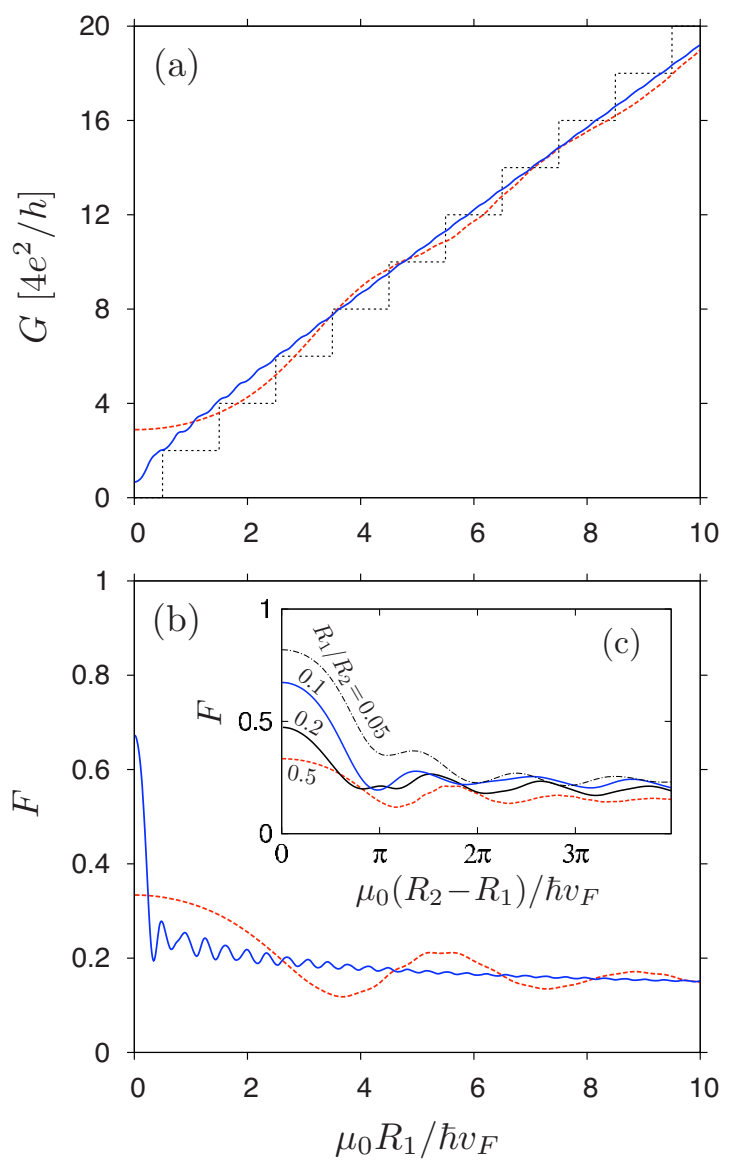

FIG. 5. (Color online) Chemical-potential dependence of the conductance (a) and Fano factor [(b) and (c)] at a fixed radii ratio $R_{1} / R_{2}$. Solid and dashed lines on panels (a) and (b) correspond to $R_{1} / R_{2}=0.1$ and 0.5 , respectively. The dotted line on panel (a) is the semiclassical approximation for the conductance. Panel (c) shows the Fano factor as a function of the chemical potential in the units of $\hbar v_{F} /\left(R_{2}-R_{1}\right)$, with $R_{1} / R_{2}$ specified for each curve on the plot.

$\pi \hbar v_{F} /\left(R_{2}-R_{1}\right)$ [for more data sets, plotted as a function of $\mu_{0}\left(R_{2}-R_{1}\right) / \hbar v_{F}=k\left(R_{2}-R_{1}\right)$, see Fig. 5(c)]. From an analytical treatment of the limit $\mu_{0} \rightarrow 0$ for angular momentum eigenstates (see Appendix B), we find that Eq. (16) for $T_{j}$ obtained within the conformal mapping technique is reproduced.

\section{Comparison with the Schrödinger system}

As the Corbino disk containing Dirac fermions described by the Hamiltonian (21) has not been studied in the literature yet, a comparison with the corresponding Schrödinger system is desirable for the sake of completeness. The existing theoretical works, ${ }^{30}$ however, focus on the model with a special angular momentum-dependent effective potential, which simplifies the analysis, but makes a relation to the Dirac system studied here unclear. For this reason, we now present a mode-matching analysis for two-dimensional nonrelativistic electron gas (2DEG) arranged in a Corbino setup with the same potential profile as applied to Dirac fermions earlier in this paper.
The Schrödinger equation for the Corbino disk in a 2DEG reads as

$$
\left[-\frac{\hbar^{2}}{2 m_{\star}} \nabla^{2}+U(r)\right] \Psi=E \Psi
$$

where $m_{\star}$ is the effective mass, and the electrostatic potential $U(r)$ is chosen identically as in the Hamiltonian (21). The solutions are written in the form of orbital-momentum $l_{z}$ eigenstates

$$
\Psi_{l}(r, \varphi)=e^{i l \varphi} \Phi_{l}(r),
$$

with $l$ integer, and the radial wave function $\Phi_{l}(r)$ a complex scalar. The propagating modes in the leads exist only for $\mu_{\infty}>0$ and have the form $\Phi_{l}^{\text {in }}(r)=H_{l}^{(2)}(K r)$ and $\Phi_{l}^{\text {out }}(r)$ $=H_{l}^{(1)}(K r)$, where $K \equiv \sqrt{2 m_{\star} \mu_{\infty} / \hbar^{2}}$, and we assume scattering from the outer lead. For the disk area, two linearly independent solutions are given by $H_{l}^{(1)}(k r)$ and $H_{l}^{(2)}(k r)$ (with $k$ $\left.\equiv \sqrt{2 m_{\star}\left|\mu_{0}\right| / \hbar^{2}}\right)$ for $\mu_{0}>0$. Otherwise, the solutions are given by modified Bessel functions $I_{l}(k r)$ and $K_{l}(k r)$. The mode-matching analysis is carried out separately for each value of $l,,^{32}$ leading to the transmission probabilities

$$
T_{l}=\frac{1}{\left|\mathfrak{M}_{l}\right|^{2}}\left(\frac{64}{\pi^{2} K^{2} R_{1} R_{2}}\right)^{2},
$$

where

$$
\begin{aligned}
\mathfrak{M}_{l}= & \mathcal{F}_{l}^{(1,1)}\left(K, k, R_{2}\right) \mathcal{F}_{l}^{(2,2)}\left(K, k, R_{1}\right) \\
& -\mathcal{F}_{l}^{(1,2)}\left(K, k, R_{2}\right) \mathcal{F}_{l}^{(2,1)}\left(K, k, R_{1}\right),
\end{aligned}
$$

$$
\begin{aligned}
\mathcal{F}_{l}^{(i, j)}(K, k, r)= & {\left[H_{l-1}^{(i)}(K r)-H_{l+1}^{(i)}(K r)\right] \mathcal{C}_{l}^{(j)}(k r) } \\
& -(k / K) H_{l}^{(i)}(K r)\left[\mathcal{C}_{l-1}^{(j)}(k r) \mp \mathcal{C}_{l+1}^{(j)}(k r)\right],
\end{aligned}
$$

with $i, j=1,2$, and the upper (lower) sign corresponding to $\mu_{0}>0\left(\mu_{0}<0\right)$. We further define

$$
\begin{gathered}
\mathcal{C}_{l}^{(1)}=\Theta\left(\mu_{0}\right) H_{l}^{(1)}+\Theta\left(-\mu_{0}\right) I_{l}, \\
\mathcal{C}_{l}^{(2)}=\Theta\left(\mu_{0}\right) H_{l}^{(2)}+\frac{4}{\pi}(-)^{l} \Theta\left(-\mu_{0}\right) K_{l},
\end{gathered}
$$

with the step function $\Theta(x)=1$ for $x>0$ or $\Theta(x)=0$ otherwise.

Numerical values of the conductance and the Fano factor following from Eq. (29) are presented in Fig. 6 (Ref. 33) for a large but finite value of the doping in the leads, adjusted such that $\sqrt{2 m_{\star}\left(U_{0}-U_{\infty}\right)} R_{1} / \hbar=7$. Both $G$ and $F$ are plotted as functions of $k\left(R_{2}-R_{1}\right)=\sqrt{2 m_{\star} \mu_{0}}\left(R_{2}-R_{1}\right) / \hbar$ for fixed values of the radii ratio $R_{1} / R_{2}=0.1,0.2$, and 0.5 (solid, dashed, and dash-dot lines, respectively); $G$ is additionally rescaled by a factor $\left(R_{2}-R_{1}\right) / R_{1}$ to illustrate its asymptotic behavior for $k R_{1} \gg 1$, which is insensitive to the ratio $R_{1} / R_{2} \cdot{ }^{34} \mathrm{We}$ also limit the discussion to $\mu_{0}>0$, as the probabilities $T_{l}$ given by Eq. (29) decay rapidly for $\mu_{0}<0$, due to the lack of propagating modes in the sample area.

Although the values of $G$ shown in Fig. 6(a) are close to the semiclassical result, ${ }^{34}$ the quantization steps are absent in the data. Instead, we observe Fabry-Pérot oscillations with the amplitude increasing with $R_{2} / R_{1}$, for either $G$ or $F$. We 


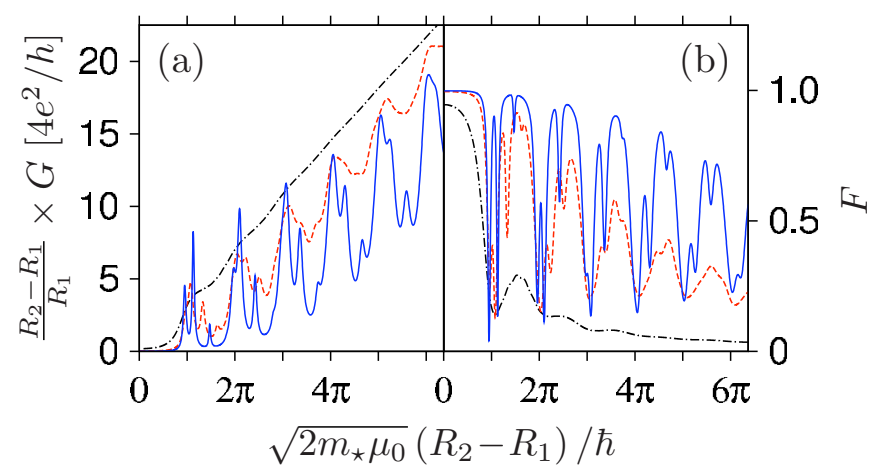

FIG. 6. (Color online) Chemical-potential dependence of the (a) conductance and the (b) Fano factor for the Corbino disk in a 2DEG. Different lines in each panel correspond to different values of the radii ratio $R_{1} / R_{2}=0.1$ (solid blue lines), 0.2 (dashed red lines), and 0.1 (black dash-dotted lines). The electrostatic potential step is fixed at $\sqrt{2 m_{\star}\left(U_{0}-U_{\infty}\right)} R_{1} / \hbar=7$.

attribute the conductance quantization reported in earlier works $^{30}$ to the particular choice of the effective radial potential (note that the existing experiments for the Corbino disk in a $2 \mathrm{DEG}^{35}$ found no conductance quantization). The main difference in transport through the Corbino disk geometry between massless fermions in graphene and massive fermions in a $2 \mathrm{DEG}$ is the reduced backscattering at the contacts and the absence of details of the leads (i.e., the doping) in the former case. ${ }^{36}$ This is a direct consequence of the energyindependent velocity in graphene, which is also responsible for the Klein-tunneling phenomena. ${ }^{37}$ Moreover, we note a suppression of the Fabry-Pérot oscillations for the relativistic system.

\section{QUANTUM BILLIARDS BOUNDED WITH SMOOTH EDGES}

\section{A. Section of the disk and circular quantum dot}

A simple generalization of the formula (14) leads to the conformal transformation that changes a finite section of the Corbino disk with the inner radius $R_{1}$, the outer radius $R_{2}$, and the spanning angle $\vartheta$ [shown in Fig. 1(b)] into a rectangle of the width $\mathcal{W}$ and the length $\mathcal{L}$, which is given by

$$
z=\frac{\mathcal{W}}{\vartheta} \log \frac{w}{R_{1}} .
$$

For $z=x+i y$, where $0 \leq x \leq \mathcal{L}$ and $0 \leq y \leq \mathcal{W}$, we get $R_{1}$ $\leq|w| \leq R_{2}$ and $0 \leq \arg w \leq \vartheta$ (with $0<\vartheta<2 \pi$ ), under the condition that $R_{2} / R_{1}=e^{\vartheta \mathcal{L} / \mathcal{W}}$. Using Eq. (7), such a condition leads to the functional $\Lambda\{z(w)\}$ in the form

$$
\Lambda=\Lambda\left(R_{1}, R_{2}, \vartheta\right)=\left(\frac{R_{2}}{R_{1}}\right)^{\pi / \vartheta} .
$$

Thus, substituting Eq. (34) into Eq. (8) leads to the exact expression for the conductance of a section of the Corbino disk. Notice that the transmission probabilities $T_{j}$ for the full disk (16) are not reproduced for $\vartheta=2 \pi$, as the mass confinement is now present in the system. Instead, they are equal for $\vartheta=\pi$, causing the conductance of such a half-disk to be equal to half of the full disk conductance for arbitrary $R_{1} / R_{2}$. The pseudodiffusive limit $\ln \Lambda \ll 1$ is realized for $R_{1} \approx R_{2}$, and the conductance (10) is

$$
G \approx G_{\mathrm{diff}}=\frac{\sigma_{0} \vartheta}{\ln \left(R_{2} / R_{1}\right)} .
$$

The above formula coincides with Eq. (17) for $\vartheta=2 \pi$. The opposite quantum-tunneling limit $(\ln \Lambda \gg 1)$ is reached for $R_{1} \ll R_{2}$, where formula (13) gives

$$
G \approx 4 \pi \sigma_{0}\left(\frac{R_{1}}{R_{2}}\right)^{\pi / \vartheta}
$$

In this case, the conductance decays (at fixed $R_{1}$ ) with the characteristic length $L \approx R_{2}$ as $G \propto L^{-\pi / \vartheta}$. The reciprocal decay observed in Sec. III for the full disk now appears at $\vartheta$ $=\pi$.

As a next example, we consider the conformal transformation, which changes the quantum dot shown in Fig. 1(c) into a rectangle of the width $\mathcal{W}$ and the length $\mathcal{L}$. The transformation is given by the formula ${ }^{25}$

$$
z-z_{0}=\frac{\mathcal{W}}{\vartheta_{\infty}} \log \frac{w+r}{w-r},
$$

with the condition $\left(R_{2}-R_{1}+r\right)^{2} /\left(R_{2}-R_{1}-r\right)^{2}=e^{\vartheta_{\infty} \mathcal{L} / \mathcal{W}}$, which leads to

$$
\Lambda\left(R_{1}, R_{2}, \vartheta\right)=\left(\frac{r-R_{1}+R_{2}}{r+R_{1}-R_{2}}\right)^{2 \pi / \vartheta_{\infty}} .
$$

The origin of the coordinate system of Fig. 2 is now shifted to $z_{0} \equiv(\mathcal{L}+i \mathcal{W}) / 2$. The poles of the transformation [marked by white dots in Fig. 1(b)] are placed at $w= \pm r$, with $r$ $\equiv \sqrt{R_{2}^{2}-R_{1}^{2}}$. The angle $\vartheta_{\infty}=\vartheta_{\infty}\left(R_{1}, R_{2}, \vartheta\right)$, at which the dot edges intersect each other, is

$$
\begin{array}{r}
\vartheta_{\infty}=2 \pi \Theta\left(\vartheta-\vartheta_{0}\right)-\operatorname{sgn}\left(\vartheta-\vartheta_{0}\right) \xi\left(\vartheta, \vartheta_{0}\right), \\
\text { with } \xi\left(\vartheta, \vartheta_{0}\right)=2 \arcsin \left(\frac{\sin \frac{\vartheta}{2} \sin \frac{\vartheta_{0}}{2}}{1-\cos \frac{\vartheta}{2} \cos \frac{\vartheta_{0}}{2}}\right),
\end{array}
$$

and $\vartheta_{0} \equiv 2 \arccos \left(R_{1} / R_{2}\right)$. Again, substituting Eq. (38) into Eq. (8) provides one with the exact expression for the system conductance, which reaches the pseudodiffusive limit for $R_{1} \approx R_{2}$, where $G \approx G_{\text {diff }}[$ see Eq. (10)] and

$$
G_{\mathrm{diff}}=\frac{\sigma_{0} \vartheta_{\infty}}{\ln \left[\left(R_{2}-R_{1}+r\right)^{2} /\left(R_{2}-R_{1}-r\right)^{2}\right]},
$$

whereas for the quantum-tunneling limit $R_{1} \ll R_{2}$, the formula (13) reads as

$$
G \approx 4 \pi \sigma_{0}\left(\frac{r+R_{1}-R_{2}}{r-R_{1}+R_{2}}\right)^{2 \pi / \vartheta_{\infty}} .
$$

This leads to an asymptotic form $G \sim\left(R_{1} / R_{2}\right)^{2 \pi / \vartheta}$, as $\vartheta_{\infty}$ $\rightarrow \vartheta$ and $r \rightarrow R_{2}$ for $R_{1} / R_{2} \rightarrow 0$, while the Fano factor approaches the Poissonian value $F \approx 1$. 

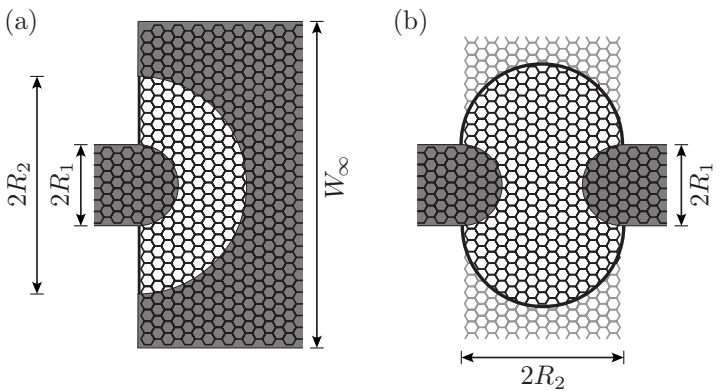

FIG. 7. (a) The half-Corbino disk and quantum dot with (b) circular edges realized on a honeycomb lattice. Shadow areas mark heavily doped graphene leads. Thick black lines indicate the mass confinement.

\section{B. Numerical results}

We now test the analytical predictions reported earlier in this section by comparing them with the results of a computer simulation of electron transport in graphene. The discussion starts from the tight-binding model of graphene, with Hamiltonian

$$
H=\sum_{i, j} \tau_{i j}|i\rangle\left\langle j\left|+\sum_{i} V_{i}\right| i\right\rangle\langle i|
$$

The hopping matrix element $\tau_{i j}=-\tau$ if the orbitals $|i\rangle$ and $|j\rangle$ are nearest neighbors on the honeycomb lattice (with $\tau$ $=2.7 \mathrm{eV}$ ), otherwise, $\tau_{i j}=0$. The single-particle potential $V_{j}$ is arranged such that the chemical potential $\mu_{j} \equiv E_{F}-V_{j}$ $=\mu_{\infty}$ in the leads marked by shadow areas in Fig. 7, whereas between the leads (white area) $\mu_{j}=0$, except for the small regions, where we put $\mu_{j}=\mu_{A, B}$ (with $\mu_{A}=-\mu_{B}$, depending whether the atom belongs to the $A$ or $B$ sublattice) to model a mass confinement on a honeycomb lattice. ${ }^{13}$ Such regions are the outermost edge atoms in the case of the half-Corbino disk [Fig. $7(\mathrm{a})]$ and the atoms placed out of the dot edge [thick lines in Fig. 7(b)] for the case of a quantum dot with circular edges.

We have calculated the transmission matrix numerically by adapting the method developed by Ando for a square lattice $^{38}$ to the honeycomb lattice. The results of our computer simulation ${ }^{39}$ depicted by data points in Fig. 8 match theoretical predictions (solid blue lines) as long as the number of modes in the narrow lead $N_{1} \gtrsim 20$. Moreover, the formulas (35) and (41) for the pseudodiffusive conductance [dashed red lines in Figs. 8(a) and 8(b)] reproduce the full expression (8) with $1 \%$ accuracy for $R_{1} \geq 0.29 R_{2}$ in the case of the half-Corbino disk, and for $R_{1} \geq 0.69 R_{2}$ in the case of the quantum dot with circular edges. Analogously, the pseudodiffusive value of the Fano factor $F \approx 1 / 3$ [see Figs. 8(c) and 8(d)] matches the full expression (9) with $1 \%$ accuracy for $R_{1} \geq 0.43 R_{2}$ and $R_{1} \geq 0.81 R_{2}$, respectively.

In other words, the half-Corbino disk, attached to one narrow and one wide lead, represents the case in which electron transport demonstrates the pseudodiffusive character in a surprisingly wide range of the system's geometrical parameters. On the contrary, in the case of the circular quantum dot attached to two narrow leads, both the conductance and the shot noise show strong deviations from the pseudodiffusive

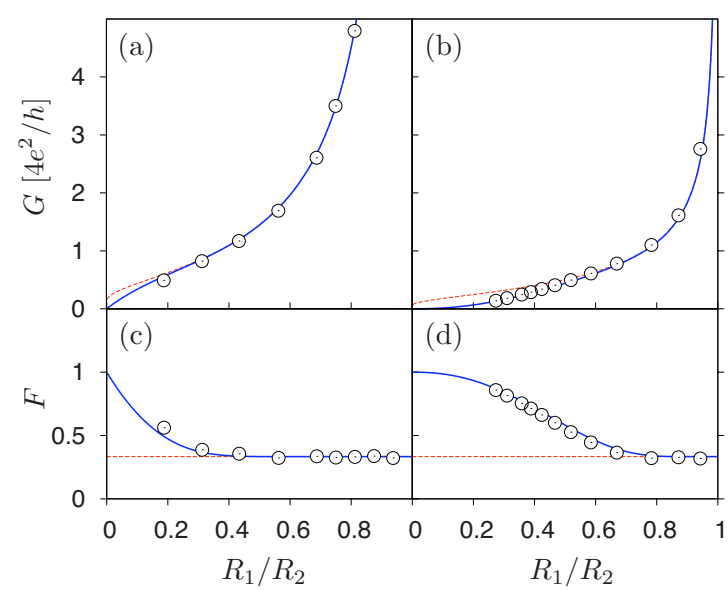

FIG. 8. (Color online) [(a) and (b)] Conductance and [(c) and (d)] Fano factor for the half-Corbino disk (left) and circular quantum dot (right). Solid lines show the results obtained by numerical summation of Eqs. (8) and (9) over the modes, dashed lines show the pseudodiffusive limits (10) and (12). Data points on left/right panels are obtained from a computer simulation of transport through the system of Figs. 7(a) and 7(b).

predictions, as the transport is dominated by a single mode in a relatively wide range of parameters. The latter represents an example of a graphene system for which our predictions on quantum-tunneling transport (such as an approximately quadratic decay of the conductance with $\left.R_{2} / R_{1}\right)$ seem to be particularly feasible for an experimental verification also because similar systems have already been fabricated, ${ }^{40}$ suggesting that the role of mass confinement is crucial when discussing the electronic structure of closed quantum dots in graphene. Moreover, a recent numerical study shows that the mass confinement leads to a strong suppression of weak localization in such systems, ${ }^{41}$ as observed earlier in experiment. $^{42}$

Below, we extend our numerical analysis to open systems that cannot be obtained from a strip by conformal transformation to illustrate the generic character of the quantumtunneling transport in undoped graphene.

\section{ELECTRON TRANSPORT ACROSS A LONG NANORIBBON}

In this section, we present the results obtained from computer simulations of transport across a long nanoribbon attached to the semicircular [Fig. 9(a)] and rectangular [Fig. 9(b)] leads, which demonstrate a striking analogy between these systems and the circular quantum dot studied in the previous section.

Each of the systems in Fig. 9 is modeled by the tightbinding Hamiltonian (43). The simulation parameters ${ }^{43}$ are chosen to grasp the basic features of recently fabricated graphene nanoribbons, ${ }^{44}$ which have zigzag edges and are insulating, as the weak staggered potential placed at the ribbon edge opens a band gap in the electronic spectrum. ${ }^{45} \mathrm{~A}$ similar effect was observed in recent numerical studies of long nanoribbons with weak edge disorder ${ }^{46}$ or irregular edges. $^{47}$ 

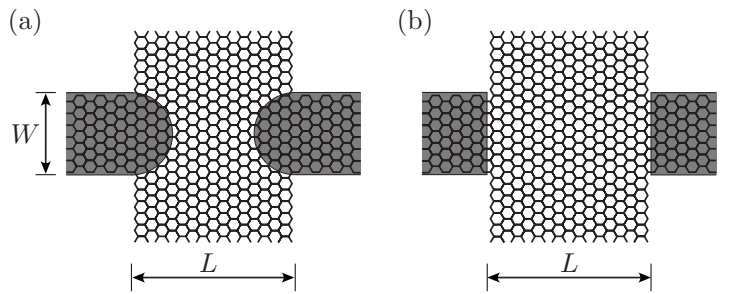

FIG. 9. Nanoribbon attached perpendicularly to the (a) semicircular and (b) rectangular leads. Each system is characterized by the lead width $W$ and the sample area length $L$. Shadow areas mark heavily doped graphene leads.

\section{A. Results for an infinitely long ribbon}

We utilize the four-terminal recursive Green's function algorithm, ${ }^{16}$ which allows us to analyze directly the electron transport across an infinitely long nanoribbon in graphene. Namely, we attached two extra leads (one from the top and one from the bottom, not shown) to each of the systems in Fig. 9 that are undoped and thus contain the evanescent modes only. (Notice that the chemical potential for the outermost edge atoms $\mu_{A, B} \neq 0 .{ }^{43}$ ) The results are shown in Fig. 10.

The conductance of a nanoribbon attached perpendicularly to circular leads (top panel in Fig. 10, open symbols) approaches the asymptotic formula for the circular quantum dot (41) with $R_{1}=W / 2, R_{2}=L / 2$, and $\vartheta=\pi$ for $W \approx L$ (solid red line). For instance, a $2 \%$ agreement is reached at $W / L$ $=0.85$. This is a consequence of the fact that in the absence of propagating modes in a ribbon, most of the current flows via the central region of the device, and the system of Fig. 9(a) becomes effectively identical to the circular quantum dot in the pseudodiffusive limit, where the role of boundary conditions is negligible. For the opposite quantum-tunneling limit $W \ll L$, the corresponding formula (42) may be written as

$$
G \approx 2^{\eta} \pi \sigma_{0}(W / L)^{2-\eta}, \quad \text { with } \quad \eta \equiv 4 W / \pi L,
$$

which agrees surprisingly well with the actual data shown in Fig. 10 (see the inset in the top panel; solid red line and open symbols, respectively). Such an agreement can be understood when looking at the current-density distribution shown in Fig. 11. Even for an aspect ratio as small as $W / L \approx 0.5$, over $90 \%$ of the current does not leave the area of a circular quantum dot (bounded symbolically with dashed lines).

For the case of a nanoribbon attached perpendicularly to rectangular leads [Fig. 9(b)], the pseudodiffusive conductance (for $W \gg L$ ) is given by ${ }^{48}$

$$
G_{\text {diff }}=\frac{\sigma_{0} W}{2 L} \frac{\pi}{\arctan \left(\frac{W}{L}\right)+\left(\frac{W}{L}\right) \ln \sqrt{1+\left(\frac{L}{W}\right)^{2}}},
$$

which is depicted in the top panel of Fig. 10 (dashed blue line) and matches the numerical data (solid symbols) within $2 \%$ accuracy for $W / L \gtrsim 2$. An identically good agreement with the numerics is observed for the asymptotic form of the formula (45) $G_{\text {diff }} \approx \sigma_{0}(W / L+1 / \pi)$, showing that the infinite ribbon attached perpendicularly to the leads has an extra
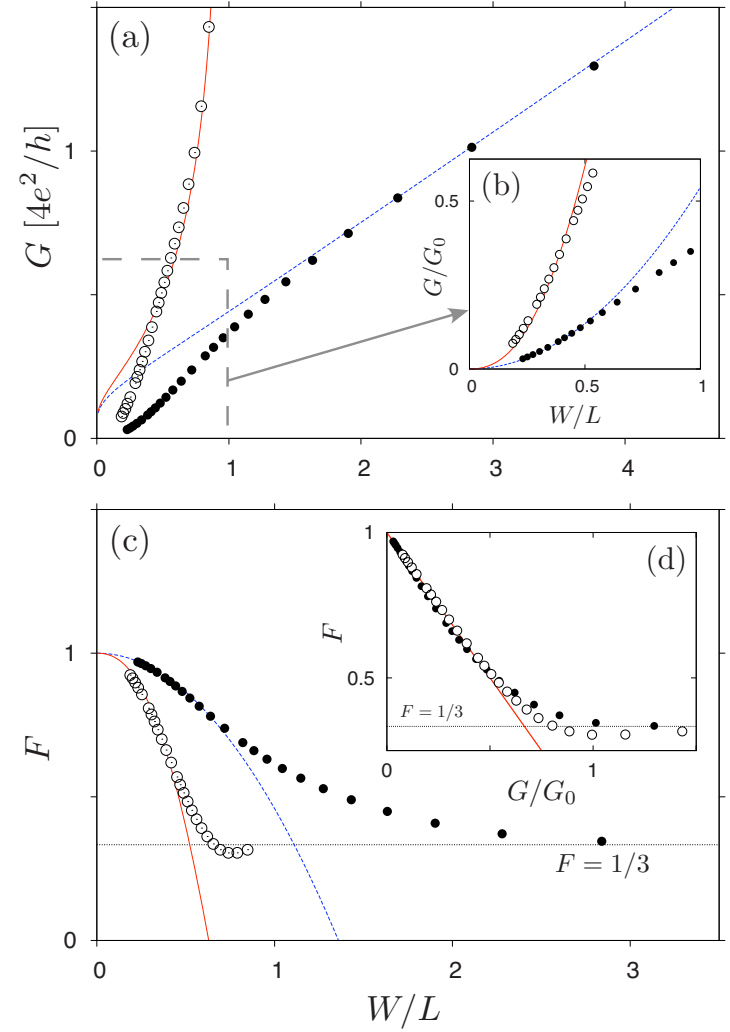

FIG. 10. (Color online) [(a) and (b)] Conductance and [(c) and (d)] Fano factor obtained numerically for the system of Figs. 9(a) and 9(b) (open and solid symbols in all panels) compared with analytical predictions (lines). (a) The pseudodiffusive conductances (41) (solid red line) and (45) (dashed blue line). Solid and dashed lines in panels (b) and (c): the tunneling conductances (44) and (47) and the corresponding values of the Fano factor $F \approx 1-G h / 4 e^{2}$. [The relation depicted by the solid line in the shot noise vs conductance diagram (d).] The pseudodiffusive limit $F=1 / 3$ is shown by the $[(\mathrm{c})$ and $(\mathrm{d})]$ black dotted line.

$\sigma_{0} / \pi$ conductance in comparison with the rectangular geometry considered in Refs. 7-10.

A brief comparison between the formula (45) and the generic form of the pseudodiffusive conductance (10) allows us
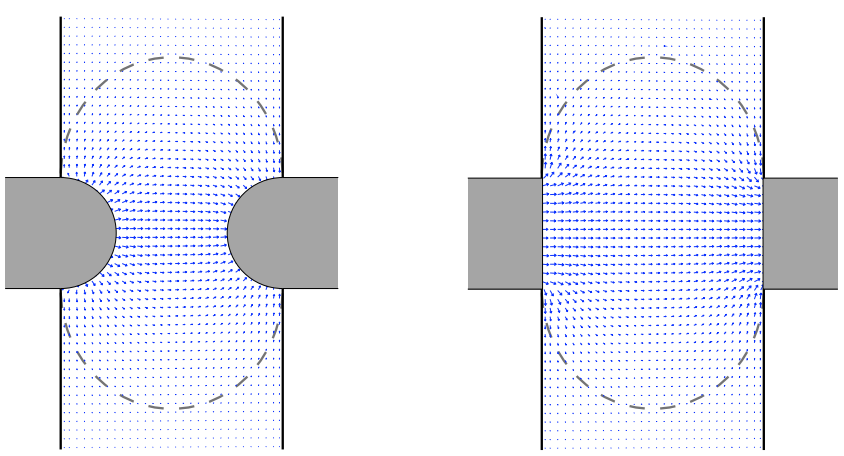

FIG. 11. (Color online) Current distribution in a long nanoribbon attached to circular (left) and rectangular (right) leads, as shown in Figs. 9(a) and 9(b). Each arrow represents the average current density for a rectangle consisting of $17 \times 17$ unit cells. The aspect ratio of both systems is $W / L \approx 0.5$. Dashed lines mark symbolically the edges of the corresponding quantum dot of Fig. 7(b). 
to consider the functional $\Lambda \equiv \Lambda(W / L)$ in an approximate form given by

$$
\ln \Lambda \approx 2\left[\ln \left(\frac{W}{L}\right)+\left(\frac{L}{W}\right) \arctan \left(\frac{W}{L}\right)\right] .
$$

Subsequently, an approximate form of the quantumtunneling conductance (13) for $W \ll L$ is

$$
G \approx 4 \pi \sigma_{0} \Lambda^{-1} \approx 4 \pi \sigma_{0} e^{-2}(W / L)^{2} .
$$

Again, the formula (47) shows a surprisingly good approximately $10 \%$ agreement with the numerical data presented in Fig. 10 (see the inset in the top panel; dashed blue line and solid symbols, respectively), suggesting that the power-law (approximately quadratic) decay of $G$ for large $L$ is a generic feature for transport across the nanoribbon unrelated to the particular shape of the leads. ${ }^{49}$

The numerical results for the shot-noise power are presented in the bottom panel of Fig. 10. The approximative formulas (44) and (47) are substituted in the relation $F \approx 1$ $-G h /\left(4 e^{2}\right)$, which produces the analytical predictions depicted by solid red and dashed blue lines, respectively. In both cases, the agreement with numerical results is better than $5 \%$ when the Fano factor $F \gtrsim 2 / 3$. An additional insight into the nature of the crossover from the Poissonian to the pseudodiffusive regime is provided by an $F$ versus $G$ plot (see the inset). In particular, values of $F$ are very close to $1-G h /\left(4 e^{2}\right)$ even for a relatively large conductance $G$ $\approx 2 e^{2} / h$, which indicates that electron transport is governed by a single valley-degenerated mode in a wide range of the geometrical parameters $(W / L \leqq 0.5$ for the circular leads, and $W / L \leqq 1.5$ for the rectangular leads).

\section{B. Influence of armchair edges in a finite ribbon}

So far, we have analyzed the transport across an infinitely long zigzag nanoribbon attached perpendicularly to the leads. To find out how the results change for the realistic case of a long but finite nanoribbon, we consider now the system of Fig. 9 with a central (undoped) region of finite width $W_{\infty}$. The system is terminated from the top and the bottom by armchair boundaries, which mix valley degrees of freedom, ${ }^{7}$ so the fourfold (spin and valley) degeneracy of transmission eigenvalues $T_{j}$ is expected to be replaced by the twofold (spin only) degeneracy. To trace the effect of armchair boundaries in a quantitative manner, we define the modeparticipation ratio

$$
P_{m}=\frac{\left(\sum_{j} T_{j}\right)^{2}}{\sum_{j} T_{j}^{2}}=\frac{2}{1-F} \frac{G}{\pi \sigma_{0}},
$$

where we assume spin-only degeneracy in summations. In particular, for the quantum-tunneling limit $W \ll L$, the modeparticipation ratio is $P_{m} \approx 2$ if the lowest mode, which governs the electronic transport, has an approximate valley degeneracy. Otherwise, in this limit $P_{m} \approx 1$.

The numerical values of the mode-participation ratio (48) are presented in Fig. 12. We took $W=80 a$ (providing 30 propagating modes for $\mu_{\infty}=\tau / 2$ ), $L=150 \sqrt{3} a$ (so $W / L \approx 0.3$ ),

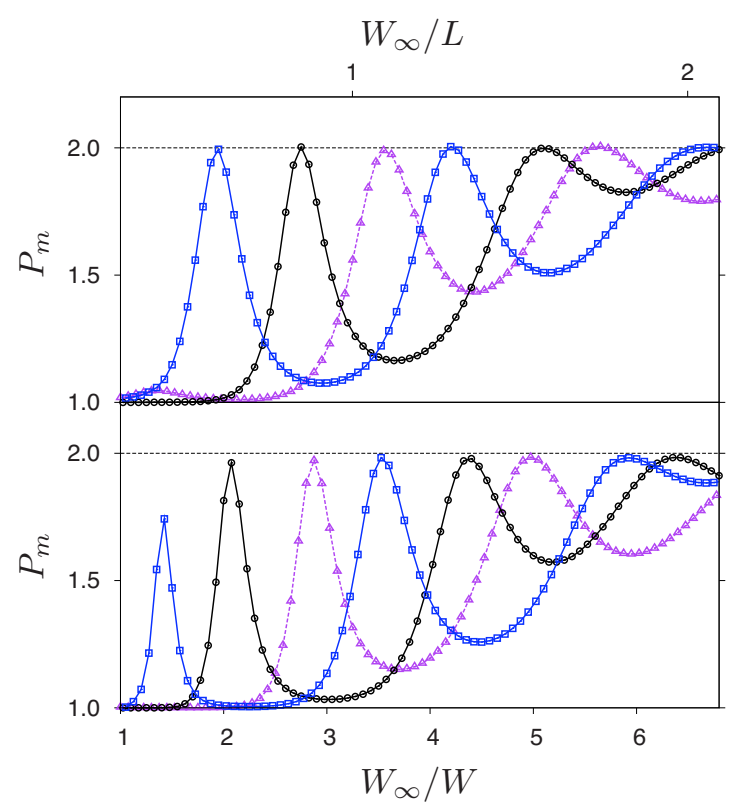

FIG. 12. (Color online) Mode-participation ratio for transport through the system of Fig. 9 with $W / L \approx 0.3$ and finite width of the undoped region $W_{\infty}$. Three curves on each panel correspond to $W_{\infty} / a=3 k$ (squares), $3 k+1$ (circles), and $3 k+2$ (triangles), with $k$ integer. Top: circular leads; bottom: rectangular leads. Lines are drawn as a guide for the eyes only.

and vary $W_{\infty}$. The remaining parameters are identical as in the case of an infinite ribbon studied before. The data points in Fig. 12 illustrate a smooth crossover from the transport dominated by a single mode with spin-only degeneracy $\left(W_{\infty} \sim W \ll L\right)$, to the situation with full fourfold degeneracy $\left(W \ll L \ll W_{\infty}\right)$. The details of the evolution depends on whether the width $W_{\infty}$ corresponds to the metallic $\left(W_{\infty} / a\right.$ $=3 k+1)$ or to one of the two insulating armchair boundary conditions $\left(W_{\infty} / a=3 k, 3 k+2\right)$. In all cases, the valley degeneracy is approximately restored $\left(P_{m} \approx 2\right.$, marked with thin black line) for $W_{\infty} \geqslant 2 L$, when the role of armchair edges becomes negligible, as the current is flowing predominantly via the central area of the system (see the current distribution shown in Fig. 11, right panel).

\section{Implications for the experiment}

For the sake of completeness, we analyze now the transport through a graphene billiard attached to two different leads, one narrow and semicircular, and the other wide and rectangular, as shown in Fig. 13. As before, mass confinement (thick black lines) is applied for the edges not connected to the leads (shadow areas). The system shown in the left panel of Fig. 13 can be exactly mapped onto a strip (see Fig. 2) by the conformal transformation (37) with the condition $\left(l^{\prime}+2 L-W\right) /\left(l^{\prime}-2 L+W\right)=e^{\vartheta_{\infty} \mathcal{L} / \mathcal{W}}$, where $l^{\prime}$ $\equiv \sqrt{4 L^{2}-W^{2}}$. This implies the functional

$$
\Lambda(W / L)=\left(\frac{l^{\prime}+2 L-W}{l^{\prime}-2 L+W}\right)^{\pi / \vartheta_{\infty}},
$$

where $\vartheta_{\infty}=2 \pi-2 \arcsin \left[l^{\prime} /(2 L)\right]$ is the angle with which edges intersect each other in the poles of conformal transfor- 


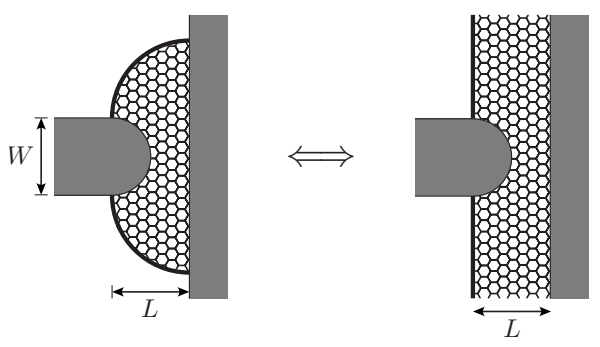

FIG. 13. These two graphene billiards both have the same conductance and shot noise in the pseudodiffusive regime $W \leqq 2 L$.

mation [see Fig. 1(c), with $\vartheta=\pi$ ]. The pseudodiffusive (10) and the quantum-tunneling conductance now take on the forms

$$
G_{\text {diff }}=\frac{\sigma_{0} \vartheta_{\infty}}{\ln \left[\left(l^{\prime}+2 L-W\right) /\left(l^{\prime}-2 L+W\right)\right]},
$$

with $\vartheta_{\infty} \rightarrow 2 \pi$ for $W / L \rightarrow 1$, and

$$
G \approx 4 \pi \sigma_{0}\left(\frac{l^{\prime}-2 L+W}{l^{\prime}+2 L-W}\right)^{\pi / \vartheta_{\infty}} \approx \pi \sigma_{0} \frac{W}{L},
$$

where the last asymptotic expression refers to the limit $W / L \rightarrow 0$, for which the system behaves effectively like the half-Corbino disk with the inner radius $R_{1}=W / 2$ and the outer radius $R_{2}=2 L$. As a consequence, the pseudodiffusive conductance (50) reproduces the values obtained from the exact expression ${ }^{50}$ with $1 \%$ accuracy for $W / L \geq 0.82$.

Another striking feature of the pseudodiffusive regime $W \leqslant 2 L$, which coincides with findings presented earlier in this section, is related to the fact that poles of the conformal transformation (37) approach the circular lead tip for $W / L$ $\rightarrow 2$. This is why the current is flowing mainly through the central area of the system and the two billiards shown in Fig. 13 become equivalent in such a limit. The earlier findings for the circular quantum dot and the long nanoribbon attached perpendicularly to the leads allow us to expect that the conductance of the nanoribbonlike system shown in the right panel of Fig. 13 will not deviate significantly from the expression $^{50}$ also in the tunneling limit $W \ll L$. The numerical results presented in Fig. 14 confirm such an expectation, as the conductance obtained by a computer simulation for the nanoribbonlike system ${ }^{51}$ match again the analytical predictions for the system with circular edges in surprisingly wide range of the parameters. Namely, $10 \%$ agreement is reached for $W / L \geq 0.4$, whereas for $W / L \geq 0.8$ the deviation drops below $2 \%$.

We predict that the pseudodiffusive conductance (50) remains unchanged for a wide class of irregular graphene billiards of shapes fitting between the two limiting cases shown in Fig. 13. Moreover, an approximate agreement should be observed even for the tunneling conductance (51). We believe that such an extra flexibility in device setups will facilitate experiments with better agreement with the theory as achieved so far for rectangular samples. ${ }^{9}{ }^{910}$ In particular, the setup consisting of the narrow semicircular lead on one side and the straight graphene-lead interface on the other side, eliminates the difficulty of manufacturing the two parallel

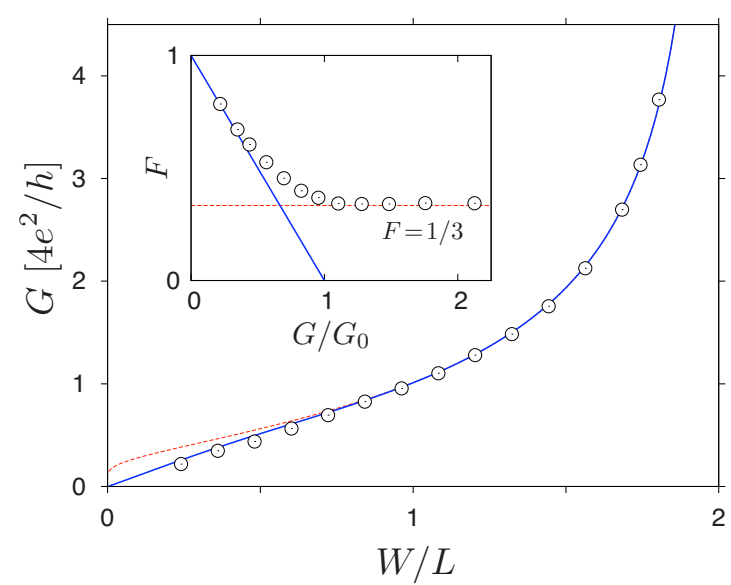

FIG. 14. (Color online) Conductance and shot noise of the two systems shown in Fig. 13. Main panel: the exact (solid line) and the pseudodiffusive (dashed line) values of conductance for a billiard with circular edges and the results of computer simulation for a nanoribbonlike billiard (datapoints). Inset: shot noise vs conductance diagram for a nanoribbonlike billiard. Solid and dashed lines mark the asymptotic values for the tunneling and the pseudodiffusive limit, respectively.

interfaces: one of the main problems that have limited the number of experimental samples, suitable for both ballistic conductance and shot-noise measurements, to just a few so far.

\section{CONCLUSIONS}

In conclusion, we have identified a type of quantumtunneling effect, which appears in transport through the Corbino disk and quantum billiards in undoped graphene provided that at least one of the leads (or billiard openings) is much narrower than the distance between openings $L$, which defines the length scale of the sample. In such a tunneling limit, the conductance $G$ shows a slow power-law decay with $L$ characterized by a geometry-dependent exponent. The Fano factor $F$ exhibits a crossover from the pseudodiffusive $(F=1 / 3)$ to Poissonian $(F=1)$ shot noise, with a relation $G$ $\approx(1-F) \times s e^{2} / h$ in a surprisingly wide range of $L$. This is because electron transport in the tunneling limit is effectively governed by a single mode, having the full spin, valley, and symplectic degeneracy $(s=8)$ in the absence of boundaries (Corbino geometry), spin and valley degeneracy $(s=4)$ if the boundary conditions do not scatter carriers between the valleys, or the spin-only degeneracy $(s=2)$ otherwise. In particular, for the case of a ribbon, which contains either infinite-mass or armchair boundaries, the valley degeneracy is restored when armchair endings are shifted away from the area where the main current flows. We would like to stress that the relation between $G$ and $F$ allows an experimental verification of the degeneracy $s$ without referring to any geometrical parameters.

We have explored the idea of Katsnelson and Guinea ${ }^{15}$ that transmission eigenvalues could be obtained analytically for any undoped graphene flake of a geometry linked via conformal transformation to a strip, for which the solution is 


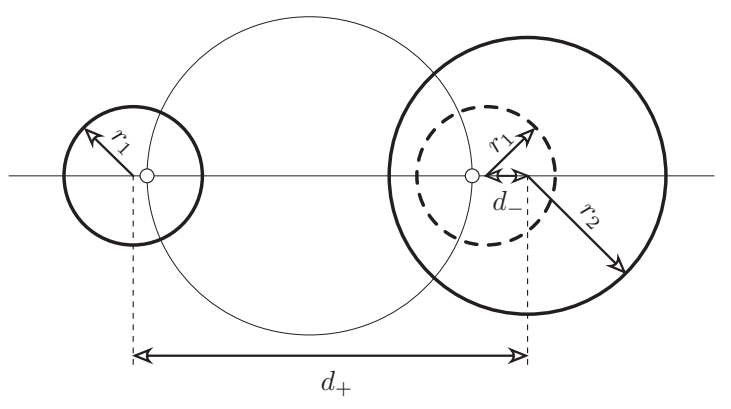

FIG. 15. Generic circular contacts (thick lines) of radii $r_{1}$ and $r_{2}>r_{1}$ misplaced by a distance $d_{+}>r_{1}+r_{2}$ or $d_{-}<r_{2}-r_{1}$ (dashed line depicts the inner contact interface for the latter case). White dots mark poles of the transformation (A1); thin lines are perpendicular to each of the interfaces (and mapped onto the radiant lines by the transformation).

known due to Tworzydło et $a .^{7}$ In the pseudodiffusive limit, we show that the eigenvalue distribution is affected by an arbitrary conformal transformation only via a multiplicative prefactor, and the value $F=1 / 3$ is unchanged for any closed setup provided that $G \gg e^{2} / h$. We test the approach for the Corbino disk, by comparing transmission probabilities obtained by a conformal mapping, and within the modematching analysis for angular momentum eigenstates. To analyze a crossover from the tunneling to the pseudodiffusive limit in a confined system, we focus on two particular billiards (a section of the Corbino disk and the quantum dot with circular edges) confined by a mass. The results of our numerical simulation of transport through a lattice consisting of approximately $10^{5}$ carbon atoms match the expressions for $G$ and $F$ obtained by conformal mapping. Moreover, we generalize the approach to obtain an approximate formula for $G$ (which reproduces either the pseudodiffusive values or the tunneling-limit exponent resulting from the simulation) in the case of an infinite ribbon attached perpendicularly to the leads: an open billiard, not linked to the strip via conformal transformation.

\section{ACKNOWLEDGMENTS}

We thank Björn Trauzettel for helpful discussions. A.R. acknowledges the support from the Alexander von Humboldt Stiftung-Foundation, the Polish Ministry of Science (Grant No. N-N202-128736), and the Polish Science Foundation (FNP). We further acknowledge financial support from the German Research Foundation (DFG) via Grants No. Tr950/ 1-1 (P.R.) and No. SFB 689 (M.W.).

\section{APPENDIX A: CONFORMAL MAPPING FOR A GENERIC SETUP WITH CIRCULAR CONTACTS}

We consider here a generic setup, containing two circular, but not coaxial interfaces splitting undoped and heavily doped graphene interfaces, as depicted schematically in Fig. 15. In the first case, an infinite graphene plane is probed by the leads of radii $r_{1}$ and $r_{2}$ (thick solid lines) misplaced by the distance $d_{+}>r_{1}+r_{2}$ (we further suppose $r_{1}<r_{2}$ ) and heavily doped. In the second case, a disklike sample area is limited by the inner lead of radius $r_{1}$ (dashed circle) and the outer lead of radius $r_{2}$ misplaced by $d_{-}<r_{2}-r_{1}$ (and for $d_{-}$ $\rightarrow 0$ the perfect Corbino geometry is restored). In both situations, conformal mapping onto the Corbino disk is provided by the Möbius transformation,

$$
z=\frac{w-\beta}{w+\beta},
$$

where $z$ belongs to the disk area (with the edges radii $R_{1}$ and $R_{2}, R_{1} \leq|z| \leq R_{2}$ ) and $w$ belongs to the sample area of Fig. 15. The real parameter $\beta$ is adjusted such that

$$
d_{ \pm}=\sqrt{r_{2}^{2}+\beta^{2}} \pm \sqrt{r_{1}^{2}+\beta^{2}},
$$

which leads to the useful relation

$$
d_{+} d_{-}=r_{2}^{2}-r_{1}^{2} \text {. }
$$

[Notice that we are using one form of $z(w)$ to describe the two distinct situations, in each of which only one of the displacements $\left\{d_{+}, d_{-}\right\}$is a physical parameter. $]$

The explicit form of the functional $\Lambda \equiv\left(R_{2} / R_{1}\right)^{1 / 2}$ follows from the condition that the transformation (A1) always maps the first contact (of radius $r_{1}$ ) onto the inner edge of the disk, whereas the second contact is mapped onto the outer edge. For the two situations studied here

$$
\Lambda\left(r_{1}, r_{2}, d_{ \pm}\right)=\left(\frac{x_{1}+y_{1}}{x_{1}-y_{1}} \frac{x_{2} \pm y_{2}}{x_{2} \mp y_{2}}\right)^{1 / 2},
$$

where we define the variables $x_{\alpha}=d_{+}+s_{\alpha} d_{-}+2 r_{\alpha}, \quad y_{\alpha}$ $=\sqrt{\left(d_{+}+s_{\alpha} d_{-}\right)^{2}-\left(2 r_{\alpha}\right)^{2}}$ (with $\left.s_{\alpha} \equiv 2 \alpha-3, \alpha=1,2\right)$. The remaining one of the parameters $\left\{d_{+}, d_{-}\right\}$not listed explicitly as an argument of $\Lambda$ is determined by Eq. (A3).

In particular, for the case of two identical circular leads probing a large graphene plane [see Fig. 4(a)] $r_{1}=r_{2} \equiv r, d_{-}$ $=0$, and the functional

$$
\Lambda\left(r, d_{+}\right)=\frac{d_{+}+2 r+\sqrt{d_{+}^{2}-4 r^{2}}}{d_{+}+2 r-\sqrt{d_{+}^{2}-4 r^{2}}} \approx \frac{d_{+}}{r},
$$

where the approximation refers to the $r \ll d_{+}$limit. Defining $l \equiv d_{+}$, we obtain Eq. (19) of the main text. Similarly, taking the limit $r_{2}, d_{+} \rightarrow \infty$ such that $l^{\prime} \equiv d_{+}-r_{1}-r_{2}=$ const, we find from Eq. (A4) that $\Lambda \approx\left(2 l^{\prime} / r\right)^{1 / 2}$ for $r \equiv r_{1} \ll l^{\prime}$, which leads to Eq. (20) for the conductance.

\section{APPENDIX B: MODE MATCHING FOR THE CORBINO DISK}

Here, we derive the transmission and reflection amplitudes for scattering eigenstates of the Hamiltonian (21) for the Corbino setup, as shown in Fig. 1(a). Without loss of generality, we suppose electron doping in the leads $E>U_{\infty}$, but an arbitrary doping in the sample area $\eta \equiv \operatorname{sgn}\left(E-U_{0}\right)$ $= \pm 1$.

The radial component $\chi_{j}(r)$ of the eigenstate $\psi_{j}(22)$ corresponding to the total angular momentum $\hbar j$ ( $j$ half-odd integer) and energy $E$ can be divided into three regions. For $r>R_{2}$ (the outer lead), $\chi_{j} \equiv \chi_{j}^{\mathrm{I}}$, with 


$$
\chi_{j}^{\mathrm{I}}=\left(\begin{array}{c}
H_{j-1 / 2}^{(2)}(K r) \\
i H_{j+1 / 2}^{(2)}(K r)
\end{array}\right)+r_{j}\left(\begin{array}{c}
H_{j-1 / 2}^{(1)}(K r) \\
i H_{j+1 / 2}^{(1)}(K r)
\end{array}\right),
$$

where $K=\left|E-U_{\infty}\right| / \hbar v_{F}$ with $U_{\infty} \rightarrow-\infty,{ }^{52}$ and $r_{j}$ the reflection coefficient. Next, for $R_{1}<r<R_{2}$ (the disk area), $\chi_{j} \equiv \chi_{j}^{\mathrm{II}}$, with

$$
\chi_{j}^{\mathrm{II}}=a_{j}\left(\begin{array}{c}
H_{j-1 / 2}^{(2)}(k r) \\
i \eta H_{j+1 / 2}^{(2)}(k r)
\end{array}\right)+b_{j}\left(\begin{array}{c}
H_{j-1 / 2}^{(1)}(k r) \\
i \eta H_{j+1 / 2}^{(1)}(k r)
\end{array}\right),
$$

where $k=\left|E-U_{0}\right| / \hbar v_{F}$. Finally, for $r<R_{1}$ (the inner lead), $\chi_{j} \equiv \chi_{j}^{\mathrm{III}}$, with

$$
\chi_{j}^{\mathrm{III}}=t_{j}\left(\begin{array}{c}
H_{j-1 / 2}^{(2)}(K r) \\
i H_{j+1 / 2}^{(2)}(K r)
\end{array}\right) .
$$

Solving the matching conditions $\chi_{j}^{\mathrm{I}}\left(R_{2}\right)=\chi_{j}^{\mathrm{II}}\left(R_{2}\right)$ and $\chi_{j}^{\mathrm{II}}\left(R_{1}\right)$ $=\chi_{j}^{\mathrm{III}}\left(R_{1}\right)$, we find

$$
a_{j}=\sqrt{\frac{2}{\pi K R_{2}}} e^{-i \kappa_{2}}\left(\eta D_{j}^{-}+i \mathfrak{D}_{j}^{+}\right)^{-1}\left[H_{j-1 / 2}^{(1)}\left(\rho_{1}\right)+i \eta H_{j+1 / 2}^{(1)}\left(\rho_{1}\right)\right],
$$

$$
b_{j}=-a_{j} \frac{H_{j-1 / 2}^{(2)}\left(\rho_{1}\right)+i \eta H_{j+1 / 2}^{(2)}\left(\rho_{1}\right)}{H_{j-1 / 2}^{(1)}\left(\rho_{1}\right)+i \eta H_{j+1 / 2}^{(1)}\left(\rho_{1}\right)},
$$

where $\rho_{\alpha}=k R_{\alpha}$ (with $\left.\alpha=1,2\right), \kappa_{2}=K R_{2}-\pi j / 2$, and we have defined

$$
\begin{aligned}
\mathfrak{D}_{j}^{ \pm}= & \operatorname{Im}\left[H_{j-1 / 2}^{(1)}\left(\rho_{1}\right) H_{j+1 / 2}^{(2)}\left(\rho_{2}\right) \pm H_{j+1 / 2}^{(1)}\left(\rho_{1}\right) H_{j \pm 1 / 2}^{(2)}\left(\rho_{2}\right)\right] \\
= & -J_{j-1 / 2}\left(\rho_{1}\right) Y_{j \mp 1 / 2}\left(\rho_{2}\right)+Y_{j-1 / 2}\left(\rho_{1}\right) J_{j \mp 1 / 2}\left(\rho_{2}\right) \\
& \mp J_{j+1 / 2}\left(\rho_{1}\right) Y_{j \pm 1 / 2}\left(\rho_{2}\right) \pm Y_{j+1 / 2}\left(\rho_{1}\right) J_{j \pm 1 / 2}\left(\rho_{2}\right), \quad
\end{aligned}
$$

with $J_{\nu}(\rho)\left[Y_{\nu}(\rho)\right]$ as the Bessel functions of the first [second] kind. The reflection and transmission amplitudes are

$$
\begin{aligned}
r_{j}(E)= & e^{-2 i \kappa_{2}}\left(\eta \mathfrak{D}_{j}^{-}+i \mathfrak{D}_{j}^{+}\right)^{-1} \\
& \times\left\{H_{j-1 / 2}^{(2)}\left(\rho_{2}\right)\left[H_{j-1 / 2}^{(1)}\left(\rho_{1}\right)+i \eta H_{j+1 / 2}^{(1)}\left(\rho_{1}\right)\right]\right. \\
& \left.-H_{j-1 / 2}^{(1)}\left(\rho_{2}\right)\left[H_{j-1 / 2}^{(2)}\left(\rho_{1}\right)+i \eta H_{j+1 / 2}^{(2)}\left(\rho_{1}\right)\right]-\eta \mathfrak{D}_{j}^{-}-i \mathfrak{D}_{j}^{+}\right\},
\end{aligned}
$$

and

$$
t_{j}(E)=\frac{4 \eta e^{i K\left(R_{1}-R_{2}\right)}}{\pi k \sqrt{R_{1} R_{2}}\left(\eta \mathfrak{D}_{j}^{-}+i \mathfrak{D}_{j}^{+}\right)} .
$$

Defining $T_{j} \equiv\left|t_{j}(E)\right|^{2}$, we obtain Eq. (24) of the main text. Notice that $T_{j}$ depends solely on $\mu_{0}=E-U_{0}$, as $t_{j}(E)$ is affected by $\mu_{\infty}=E-U_{\infty}$ only via a phase factor. It is also insensitive to the doping sign $\eta= \pm 1$, which corresponds to the particle-hole symmetry.

For the undoped-disk limit $(k \rightarrow 0)$, Eq. (B6) leads to the asymptotic form

$$
\eta \mathfrak{D}_{j}^{-}+i \mathfrak{D}_{j}^{+} \approx \frac{2 \eta}{\pi k \sqrt{R_{1} R_{2}}}\left[\left(\frac{R_{1}}{R_{2}}\right)^{j}+\left(\frac{R_{2}}{R_{1}}\right)^{j}\right] .
$$

Substituting the above formula into Eq. (B8), we obtain $T_{j}$ $=\left|t_{j}\left(E \rightarrow U_{0}\right)\right|^{2}$ as given by Eq. (16) of the main text. Hence, the correspondence between the mode matching for angular momentum eigenstates and the conformal mapping technique for the disk in undoped graphene is established.
*Present address: Instituut-Lorentz, Universiteit Leiden, P.O. Box 9506, 2300 RA Leiden, The Netherlands.

${ }^{1}$ For a review on the topic, see A. K. Geim and K. S. Novoselov, Nature Mater. 6, 183 (2007).

${ }^{2}$ Y. Imry, Introduction to Mesoscopic Physics (Oxford University Press, Oxford, 1996).

${ }^{3}$ P. R. Wallace, Phys. Rev. 71, 622 (1947).

${ }^{4}$ K. Nomura and A. H. MacDonald, Phys. Rev. Lett. 98, 076602 (2007); M. Titov, EPL 79, 17004 (2007); A. Rycerz, J. Tworzydło, and C. W. J. Beenakker, ibid. 79, 57003 (2007).

${ }^{5}$ A. Rycerz, J. Tworzydło, and C. W. J. Beenakker, Nat. Phys. 3, 172 (2007); W. Yao, S. A. Yang, and Q. Niu, Phys. Rev. Lett. 102, 096801 (2009); G. Tkachov and M. Hentschel, Phys. Rev. B 79, 195422 (2009).

${ }^{6}$ M. I. Katsnelson, Eur. Phys. J. B 51, 157 (2006).

${ }^{7}$ J. Tworzydło, B. Trauzettel, M. Titov, A. Rycerz, and C. W. J. Beenakker, Phys. Rev. Lett. 96, 246802 (2006).

${ }^{8}$ For the numerical study of a system with zigzag boundary condition, not considered in Refs. 6 and 7, see A. Rycerz, Phys. Status Solidi A 205, 1281 (2008).

${ }^{9}$ F. Miao, S. Wijeratne, Y. Zhang, U. C. Coscun, W. Bao, and C. N. Lau, Science 317, 1530 (2007).

${ }^{10}$ R. Danneau, F. Wu, M. F. Craciun, S. Russo, M. Y. Tomi, J.
Salmilehto, A. F. Morpurgo, and P. J. Hakonen, Phys. Rev. Lett. 100, 196802 (2008).

${ }^{11}$ X. Du, I. Skachko, A. Barker, and E. Y. Andrei, Nat. Nanotechnol. 3, 491 (2008).

${ }^{12}$ M. Müller, M. Bräuninger, and B. Trauzettel, arXiv:0812.4141 (unpublished).

${ }^{13}$ A. R. Akhmerov and C. W. J. Beenakker, Phys. Rev. B 77, 085423 (2008).

${ }^{14}$ For a computer simulation of transport for the Hall-bar setup used in Ref. 9, see R. Golizadeh-Mojarad and S. Datta, Phys. Rev. B 79, 085410 (2009).

${ }^{15}$ M. I. Katsnelson and F. Guinea, Phys. Rev. B 78, 075417 (2008).

${ }^{16}$ M. Wimmer and K. Richter, arXiv:0806.2739 (unpublished).

${ }^{17}$ E. B. Sonin, Phys. Rev. B 77, 233408 (2008).

${ }^{18}$ S. Datta, Electronic Transport in Mesoscopic Systems (Cambridge University Press, Cambridge, 1997).

${ }^{19}$ Here we limit the analysis to a single valley. For a general discussion, see the review by C. W. J. Beenakker, Rev. Mod. Phys. 80, 1337 (2008).

${ }^{20}$ The translational invariance along the $y$ axis is satisfied for an infinitely wide strip or for periodic boundary conditions of Ref. 6. In case of a confined geometry that does not mix the valleys, 
one can use $\Psi(x, y)=\chi_{\theta}(x) e^{i k_{y} y}+\chi_{-\theta}(x) e^{-i k_{y} y}$ for the mode matching and find that Eq. (4) remains unchanged.

${ }^{21}$ H. Schomerus, Phys. Rev. B 76, 045433 (2007); Ya. M. Blanter and I. Martin, ibid. 76, 155433 (2007).

${ }^{22}$ Zero-energy solution of the Dirac equation in two dimensions has the general form $\Psi=\left[\Psi_{A}(x+i y), \Psi_{B}(x-i y)\right]^{T}$, where the spinor components $\Psi_{A}$ and $\Psi_{B}$ are holomorphic functions. For the strip geometry, infinite-mass boundary conditions $\left(\left.\Psi_{A}\right|_{y=0}\right.$ $\left.=\left.\Psi_{B}\right|_{y=0},\left.\Psi_{A}\right|_{y=\mathcal{W}}=-\left.\Psi_{B}\right|_{y=\mathcal{W}}\right)$ are satisfied after applying the transformation $\Psi_{A}=\Psi_{A}[z(w)], \Psi_{B}=\Psi_{B}\left[z\left(w^{\star}\right)\right]$, with $z(w) \equiv x(u$ $+i v)+i y(u+i v)$ an analytical function that turns the coordinate system $(u, v)$ into $(x, y)$. The case of antiperiodic bc is discussed in Sec. III.

${ }^{23}$ In the special case of antiperiodic bc, $s=8$ for all the modes. For periodic bc considered in Ref. 6, the lowest fully transmitted mode has only a fourfold (spin and valley) degeneracy.

${ }^{24}$ O. N. Dorokhov, Solid State Commun. 51, 381 (1984).

${ }^{25}$ W. R. Smythe, Static and Dynamic Electricity, 3rd ed. (McGrawHill, New York, 1968), Chap. 4.

${ }^{26}$ P. Carmier and D. Ullmo, Phys. Rev. B 77, 245413 (2008).

${ }^{27}$ A. H. Castro Neto, F. Guinea, N. M. R. Peres, K. S. Novoselov, and A. K. Geim, Rev. Mod. Phys. 81, 109 (2009).

${ }^{28}$ V. B. Shikin, Pis'ma Zh. Exp. Teor. Fiz. 66, 545 (1997).

${ }^{29}$ P. Recher, B. Trauzettel, A. Rycerz, Ya. M. Blanter, C. W. J. Beenakker, and A. F. Morpurgo, Phys. Rev. B 76, 235404 (2007).

${ }^{30}$ G. Kirczenow, J. Phys.: Condens. Matter 6, L583 (1994); S. Souma and A. Suzuki, Phys. Rev. B 58, 4649 (1998).

${ }^{31}$ C. W. J. Beenakker, R. A. Sepkhanov, A. R. Akhmerov, and J. Tworzydło, Phys. Rev. Lett. 102, 146804 (2009).

${ }^{32} \mathrm{We}$ took $\Phi_{l}^{\mathrm{I}}(r)=H_{l}^{(2)}(K r)+r_{l} H_{l}^{(1)}(K r)$ for $r>R_{2}, \quad \Phi_{l}^{\mathrm{II}}(r)$ $=a \mathcal{C}_{l}^{(1)}(k r)+b \mathcal{C}_{l}^{(2)}(k r)$ with $\mathcal{C}_{l}^{(1,2)}$ given by Eq. (32) for $R_{2}>r$ $>R_{1}, \Phi_{l}^{\mathrm{III}}(r)=t_{l} H_{l}^{(2)}(K r)$ for $R_{1}>r$, and solved the matching conditions $\quad \Phi_{l}^{\mathrm{I}}\left(R_{2}\right)=\Phi_{l}^{\mathrm{II}}\left(R_{2}\right), \quad \Phi_{l}^{\mathrm{II}}\left(R_{1}\right)=\Phi_{l}^{\mathrm{III}}\left(R_{1}\right),\left.\quad \frac{d \Phi_{l}^{\mathrm{I}}}{d r}\right|_{R_{2}}$ $=\left.\frac{d \Phi_{l}^{\mathrm{II}}}{d r}\right|_{R_{2}},\left.\frac{d \Phi_{l}^{\mathrm{II}}}{d r}\right|_{R_{1}}=\left.\frac{d \Phi_{l}^{\mathrm{III}}}{d r}\right|_{R_{1}}$.

${ }^{33} \mathrm{We}$ use the Landauer formula $G=\left(2 e^{2} / h\right) \sum_{l=-\infty}^{\infty} T_{l}$, and $F$ $=\Sigma_{l=-\infty}^{\infty} T_{l}\left(1-T_{l}\right) / \Sigma_{l=-\infty}^{\infty} T_{l}$. Note the twofold degeneracy $T_{l}=T_{-l}$ for $l \neq 0$.

${ }^{34}$ The semiclassical conductance for nonrelativistic electrons in the Corbino disk is $G_{\mathrm{s}-\mathrm{cl}}=\left(4 e^{2} / h\right)\left(l_{1}+1 / 2\right)$ with $l_{1}=\operatorname{int}\left[\left(k^{2} R_{1}^{2}\right.\right.$ $\left.+1 / 4)^{1 / 2}\right]$ (see Ref. 30). It leads to $G \propto k R_{1}$ for $k R_{1} \gg 1$.

${ }^{35}$ R. P. Taylor, R. Newbury, A. S. Sachrajda, Y. Feng, P. T. Coleridge, M. Davies, and J. P. McCaffrey, Superlattices Microstruct. 24, 337 (1998).

${ }^{36}$ For a $2 D E G$ and the limit of $K \gg k$, we find from Eq. (29) that $T_{l} \approx 64\left(\pi^{2} K^{2} R_{1} R_{2}\right)^{-1}\left|\mathcal{C}_{l}^{(1)}\left(k R_{2}\right) \mathcal{C}_{l}^{(2)}\left(k R_{1}\right)-\mathcal{C}_{l}^{(2)}\left(k R_{2}\right) \mathcal{C}_{l}^{(1)}\left(k R_{1}\right)\right|^{-2}$, which vanishes as $(1 / K)^{2}$.

${ }^{37}$ M. I. Katsnelson, K. S. Novoselov, and A. K. Geim, Nat. Phys. 2, 620 (2006); V. V. Cheianov and V. I. Fal'ko, Phys. Rev. B 74, 041403(R) (2006); A. V. Shytov, M. S. Rudner, and L. S. Levitov, Phys. Rev. Lett. 101, 156804 (2008); N. Stander, B. Huard, and D. Goldhaber-Gordon, ibid. 102, 026807 (2009).
${ }^{38}$ T. Ando, Phys. Rev. B 44, 8017 (1991).

${ }^{39}$ For the half-Corbino disk $(\vartheta=\pi)$, we have fixed the width of the wide lead attached from the right [see Fig. 7(a)] at $W_{\infty}=560 a$ (corresponding to $N_{\infty}=213$ propagating modes for $\mu_{\infty}=\tau / 2$ ). The inner radius is varied in the range $R_{1}=30-150 a\left(N_{1}\right.$ $=23-114$ modes). The remaining parameters are the outer radius $R_{2}=160 a$ (the diameter $2 R_{2}$ is noticeably smaller than $W_{\infty}$ to model the bulk-lead regime) and the edge-atoms chemical potential $\mu_{A}=-\mu_{B}=0.01 \tau$. For the quantum dot with circular edges [see Fig. 7(b)], we have fixed the lead radius at $R_{1}=40 a\left(N_{1}\right.$ $=30$ for $\left.\mu_{\infty}=\tau / 2\right)$, the edge radius is varied in the range $24 \sqrt{3} a<R_{2}<85 \sqrt{3} a$, and the staggered-potential amplitude outside the dot is varied in the range $\left|\mu_{A, B}\right|=0.1-0.8 \tau$. We find that the results are insensitive to the confinement strength.

${ }^{40}$ L. A. Ponomarenko, F. Schedin, M. I. Katsnelson, R. Yang, E. W. Hill, K. S. Novoselov, and A. K. Geim, Science 320, 356 (2008).

${ }^{41}$ J. Wurm, A. Rycerz, I. Adagideli, M. Wimmer, K. Richter, and H. U. Baranger, Phys. Rev. Lett. 102, 056806 (2009).

${ }^{42}$ F. V. Tikhonenko, D. W. Horsell, R. V. Gorbachev, and A. K. Savchenko, Phys. Rev. Lett. 100, 056802 (2008).

${ }^{43}$ The chemical potential $\mu_{j}=0$ in the undoped region (white areas in Fig. 9), except for the outermost edge atoms, where we put $\mu_{j}=\mu_{A, B}$, with $\mu_{A}=-\mu_{B}=0.01-0.1 \tau$. In the leads (shadow areas) $\mu_{j}=\mu_{\infty}=0.4 \tau$, what corresponds to 60 propagating modes (for the lead width fixed at $W=200 a$ ). The sample area length $L$ is varied but kept equal to the width of undoped leads.

${ }^{44}$ X. Li, X. Wang, L. Zhang, S. Lee, and H. Dai, Science 319, 1229 (2008); M. Y. Han, B. Özyilmaz, Y. Zhang, and P. Kim, Phys. Rev. Lett. 98, 206805 (2007); Zh. Chen, Y.-M. Lin, M. J. Rooks, and P. Avouris, Physica E 40, 228 (2007).

${ }^{45}$ Y.-W. Son, M. L. Cohen, and S. G. Louie, Phys. Rev. Lett. 97, 216803 (2006); M. Wimmer, I. Adagideli, S. Berber, D. Tomanek, and K. Richter, ibid. 100, 177207 (2008).

${ }^{46}$ E. R. Mucciolo, A. H. Castro Neto, and C. H. Lewenkopf, Phys. Rev. B 79, 075407 (2009).

${ }^{47}$ D. Rainis, F. Taddei, F. Dolcini, M. Polini, and R. Fazio, Phys. Rev. B 79, 115131 (2009).

${ }^{48}$ We suppose the current density $\mathbf{j}=\sigma_{0} \mathbf{E}=-\sigma_{0} \nabla \varphi$, where $\varphi(x, y)$ is an electrostatic potential. The conductance $G=I /\left(\varphi_{x=L / 2}\right.$ $\left.-\varphi_{x=-L / 2}\right)$ is found by the analytical integration of the current passing the vertical symmetry axis of the system $(x=0)$.

${ }^{49}$ Reference 15 reports an exponential decay for a similar geometry, but it considers a far different leads arrangement, for which the current is effectively flowing along a narrow insulating nanoribbon in graphene.

${ }^{50}$ Equation (8) with $s=4$ and $\Lambda=\Lambda(W / L)$ given by Eq. (49).

${ }^{51}$ Parameters of the nanoribbonlike system studied numerically are $L=96 \sqrt{3} a, W_{\infty}=560 a, \mu_{\infty}=\tau / 2$, and $\left|\mu_{A, B}\right|=0.01 \tau$. The narrowlead width is varied in the range $W / a=40-300$.

${ }^{52}$ We use $H_{\nu}^{(1)}(\rho) \approx \sqrt{2 /(\pi \rho)} \exp [i(\rho-\nu \pi / 2-\pi / 4)]$ for $\rho \gg 1$, and $H_{\nu}^{(2)}(\rho)=\left[H_{\nu}^{(1)}(\rho)\right]^{\star}$. 